\title{
Perfiles y funciones del tutor universitario y sus efectos sobre las necesidades tutoriales del alumnado
}

\section{Profiles and functions of university tutors and their effects on students' tutorial needs}

\author{
Dr. Manuel DELGADO-GARCÍA. Profesor Ayudante Doctor. Universidad de Huelva (manuel.delgado@dedu.uhu.es). \\ Dra. Sara CONDE VÉLEZ. Profesora Ayudante Doctora. Universidad de Huelva (sara.conde@dedu.uhu.es). \\ Dr. Ángel BOZA CARREÑo. Profesor Titular. Universidad de Huelva (aboza@uhu.es).
}

\section{Resumen:}

La educación superior se caracteriza por una masificación de estudiantes en determinadas titulaciones y una progresiva despersonalización del proceso de enseñanza y aprendizaje. Esta realidad justifica la necesidad de analizar los procesos de acción tutorial que se desarrollan en la actualidad. Este trabajo tiene como objetivo indagar en varios factores asociados a los principales agentes implicados en dicho proceso: tutor (perfiles y funciones) y alumnado (necesidades). Se parte de una metodología tipo encuesta en la que se construye y se somete a procesos de validación un instrumento distribuido entre los estudiantes de grado de la Universidad de Huelva, con el objetivo de ob- tener un modelo teórico de referencia desde el que valorar las interacciones entre los factores emergentes.

Como resultados, cabe destacar un modelo teórico en el que existen correlaciones entre el perfil del tutor, y regresiones o influencias de estos perfiles sobre las funciones y las necesidades de los estudiantes; de la misma manera, se visibilizan dos tipologías de tutor universitario que priorizan los aspectos académicos y personales de la tutoría respectivamente. Ambos perfiles llevan asociados unos tipos de funciones que vendrán a dar respuesta a las necesidades que presentan los estudiantes a lo largo de su paso por la universidad y que

Fecha de recepción de la versión definitiva de este artículo: 18-09-2019.

Cómo citar este artículo: Delgado-García, M., Conde Vélez, S. y Boza Carreño, Á. (2020). Perfiles y funciones del tutor universitario y sus efectos sobre las necesidades tutoriales del alumnado | Profiles and functions of university tutors and their effects on students' tutorial needs. Revista Española de Pedagogía, 78 (275), 119-143. doi: https://doi. org/10.22550/REP78-1-2020-03

https://revistadepedagogia.org/ 
apoyan las tesis del avance de la tutoría universitaria hacia un modelo integral en el que la dimensión personal cobra una especial relevancia.

Descriptores: orientación educativa, tutoría, educación superior, necesidades de orientación del alumnado, perfil competencial del tutor.

\section{Abstract:}

Large numbers of students on particular degrees and a progressive depersonalisation of the teaching and learning process are typical characteristics of higher education. This situation results in a need to analyse the tutorial activity processes currently in use. This work aims to investigate various factors associated with the main agents involved in this process: tutors (profiles and functions) and students (needs). It is based on a survey-type methodology creating an instrument for distribution among degree students at the Universidad de Huelva. This is first subjected to validation processes to obtain a theoretical reference model with which to evaluate interactions among the emerging factors.

Notable results include a theoretical model with correlations between tutor profiles and regressions, or influences of these profiles on functions and student needs. Furthermore, it reveals two types of university tutor: those who prioritise the academic aspects of tutoring, and those who prioritise personal aspects. Both profiles are associated with types of function that react to the needs students display throughout their time at university, and support the thesis that university tutoring is developing towards an integral model in which the personal dimension is especially relevant.

Keywords: educational guidance, tutoring, higher education, student guidance needs, competence profile of tutors.

\section{Introducción}

En el marco de la educación superior, la acción tutorial se entiende como una función inherente a la labor del docente, empleada como un modelo de educación personalizada desde el que atender de manera integral al estudiante y que contribuye a «disminuir la ansiedad, favorecer la integración a la nueva institución y mejorar las condiciones para el aprendizaje» (Fernández-Salinero,
2014, p. 163). Junto a estos factores existen otros elementos asociados a la universidad del siglo xxI (diversidad entre el alumnado, diversificación en los currículos, incertidumbre ante el futuro y la inserción profesional, incesantes cambios sociales, formativos y profesionales) que generan la necesidad de integrar la tutoría en el currículum y ser impulsada desde las políticas institucionales (Álvarez González, 2017; 
Martínez Clares, Pérez Cusó y González Morga, 2019).

En la actualidad, son variadas las acepciones utilizadas para definir los términos acción tutorial o tutoría (tutoring action o tutoring) (Álvarez González y Álvarez, 2015; Álvarez González y Bisquerra, 2012; Álvarez-Pérez, 2014; González-Benito y Vélaz de Medrano, 2014; Hagenauer y Volet, 2014; Pantoja, 2013; García Nieto, 2011; McFarlane, 2016; Yale, 2019), de las que se derivan múltiples dimensiones de análisis. En este sentido, trabajos como los de López-Gómez (2015, 2017), León (2018) o Martínez Clares, Martínez Juárez y Pérez Cusó (2016) realizan una amplia revisión y plasman una clara prospectiva del término para atender a su compresión y evolución, de manera que pueda llegar a definirse como un proceso de índole formativa que desarrolla el docente universitario apoyándose en los presupuestos de la orientación, para atender desde un plano integral las necesidades del estudiante (académicas, sociales-personales y/o profesionales) con el objetivo de favorecer un proceso de enseñanza y de aprendizaje lo más óptimo posible.

A raíz de este concepto, se extraen tres dimensiones claramente definitorias de lo que aporta este trabajo y que están ligadas a la labor del tutor (perfil y funciones) y a las necesidades tutoriales de los estudiantes.

\section{Funciones y perfiles del profe- sor-tutor universitario}

Desde un plano normativo, el Estatuto del Estudiante Universitario (Real
Decreto 1791/2010, de 30 de diciembre), en su artículo 8e, instaura como derecho «recibir orientación y tutoría personalizada para facilitar la incorporación laboral, el desarrollo profesional y la continuidad, en su caso, de la formación universitaria» y en el artículo 20, identifica claramente que el responsable de esta labor será el profesor-tutor, quien ofrecerá una respuesta a tres niveles (en el momento de ingreso en la universidad, durante los estudios universitarios y en la transición al mundo laboral); a partir de este postulado, existen múltiples trabajos que indagan y evalúan el quehacer profesional del tutor con el objetivo de sentar las bases para una adecuada acción tutorial (Álvarez-Pérez, López y Pérez, 2016; Arza, Salvador y Mascarenhas, 2014; Bisquerra, 2013; Gaitán, 2013; García-Valcárcel, 2008; López-Gómez, 2017; Martínez Clares et al., 2016; Torrecilla, Rodríguez, Herrera y Martín, 2013; Troyano y García, 2009; Urbina, de la Calleja y Medina, 2017).

Como consecuencia de los enfoques de acción tutorial derivados de estos trabajos, compartimos la estructura de Lobato y Guerra (2016) a partir de la cual emergen siete modalidades de tutoría en la universidad, de las cuales cinco son explícitamente desarrolladas por el profesorado universitario. A su vez, siguiendo los trabajos de Rodríguez Espinar (2004), completados por Álvarez González (2013, 2014) y Álvarez González y Álvarez (2015), entendemos que las tareas del tutor universitario se engloban en base a tres modelos de acción tutorial (Tabla 1). 
TABLA 1. Modalidades de tutoría en la universidad.

\begin{tabular}{|c|c|c|c|}
\hline Modalidad & Definición de funciones & Figura & Sistema \\
\hline $\begin{array}{l}\text { Tutoría } \\
\text { académica }\end{array}$ & $\begin{array}{l}\text { Seguimiento y apoyo del proceso de aprendi- } \\
\text { zaje en una asignatura o materia (atender las } \\
\text { dificultades surgidas; flexibilizar las metodo- } \\
\text { logías de trabajo; elaborar productos de eva- } \\
\text { luación; promover el desarrollo de trabajos de } \\
\text { investigación; desarrollar una comunicación } \\
\text { efectiva; hacer uso de un comportamiento y } \\
\text { compromiso crítico, etc.). }\end{array}$ & \multirow[t]{3}{*}{ Docente } & $\begin{array}{l}\text { Plan de Ac- } \\
\text { ción Tutorial } \\
\text { (PAT) de } \\
\text { universidad o } \\
\text { de facultad }\end{array}$ \\
\hline $\begin{array}{c}\text { Tutoría } \\
\text { de Practicum }\end{array}$ & $\begin{array}{l}\text { Asesoramiento al estudiante en período de } \\
\text { prácticas en un centro profesional. }\end{array}$ & & $\begin{array}{l}\text { Programa de } \\
\text { Practicum }\end{array}$ \\
\hline $\begin{array}{c}\text { Tutoría } \\
\text { de } \\
\text { investigación }\end{array}$ & $\begin{array}{l}\text { Asesoramiento individualizado en la elabora- } \\
\text { ción de un trabajo de investigación en el gra- } \\
\text { do, el posgrado o el doctorado. }\end{array}$ & & $\begin{array}{l}\text { Programada } \\
\text { entre docente } \\
\text { y estudiante }\end{array}$ \\
\hline $\begin{array}{l}\text { Tutoría } \\
\text { personal }\end{array}$ & $\begin{array}{l}\text { Atención individualizada y especializada para } \\
\text { resolver dificultades personales que afectan al } \\
\text { rendimiento académico (ayudar al estudiante } \\
\text { en el proceso de conocimiento de sí mismo; } \\
\text { guiar en el proceso de toma de decisiones } \\
\text { personales; contribuir a la resolución de con- } \\
\text { flictos; fomentar el desarrollo de habilidades } \\
\text { sociales; etc.). }\end{array}$ & $\begin{array}{l}\text { Profesional } \\
\text { especialista } \\
\text { / Docente }\end{array}$ & $\begin{array}{l}\text { A petición del } \\
\text { estudiante }\end{array}$ \\
\hline $\begin{array}{l}\text { Tutoría } \\
\text { de titulación } \\
\text { o carrera }\end{array}$ & $\begin{array}{l}\text { Intervención de apoyo al desarrollo personal, } \\
\text { académico y profesional a lo largo de toda la } \\
\text { trayectoria universitaria del estudiante (cono- } \\
\text { cer el perfil del estudiante; atender al proce- } \\
\text { so de acogida del estudiante; contribuir a la } \\
\text { integración académico-social del estudiante; } \\
\text { facilitar información y orientación curricular, } \\
\text { etc.). }\end{array}$ & Docente & PAT \\
\hline $\begin{array}{l}\text { Tutoría entre } \\
\text { iguales }\end{array}$ & $\begin{array}{l}\text { Estudiante experimentado que apoya a com- } \\
\text { pañeros de titulación. }\end{array}$ & Estudiante & PAT \\
\hline $\begin{array}{l}\text { Tutoría } \\
\text { de servicio }\end{array}$ & $\begin{array}{l}\text { Atención de información y asesoramiento aca- } \\
\text { démico y laboral a todo el alumnado de la uni- } \\
\text { versidad. }\end{array}$ & Técnicos & $\begin{array}{c}\text { Servicio } \\
\text { institucional }\end{array}$ \\
\hline
\end{tabular}

Fuente: Adaptación de Lobato y Guerra (2016, p. 385).

Para el desarrollo de estas funciones, García Cabrero et al. (2016) sugieren que el tutor tiene que adquirir capacidades a la vez que ser conocedor de la información institucional, puesto que de este grado de profesionalización va a depender en gran medida ofrecer una respuesta acorde a las necesidades que demanda el estudiante. Así pues, es necesario tomar en consideración el rol que adopte el profesorado universitario (actitudes) con respecto a la importancia atribuida a la acción tutorial, entendiendo claramente, como afirma Más Torelló (2012, p. 303), que «tutorizar el proceso de aprendizaje del alumno propiciando acciones que le permitan una ma- 
yor autonomía» ha de ser una de las competencias básicas de su perfil profesional.

A este respecto, la investigación educativa (Fernández-Salinero, 2014; González-Benito y Vélaz de Medrano, 2014; González-Lorente y González-Morga, 2015; Herrera Rodríguez, 2017; López, González y Velasco, 2013; Martínez Clares, Martínez Juárez y Pérez Cusó, 2014; Martínez-Mínguez y Moya, 2017) ha logrado identificar rasgos compartidos al afrontar esta función y contribuir en la configuración de perfiles profesionales reconocibles y con atribuciones competenciales concretas que integrarán «ciertas habilidades, conocimientos adecuados y una actitud determinada, guiada por principios éticos» (Giner, 2012, p. 28). Esto configura un amplio espectro de formas de actuación respecto al ejercicio de la acción tutorial en la universidad, sintetizadas en el modelo paternalista (pastoral care) y el modelo más distante (laissez-faire) como extremos desde los que asumir diferentes grados de implicación, acción y compromiso (Cid y Pérez, 2006).

En líneas generales, siguiendo los trabajos de Echeverría (2002), García Nieto, Asensio Muñoz, Carballo Santaolalla, García García y Guardia González (2004), y Lázaro (2003), podría establecerse una clasificación que atiende a los ámbitos de acción del tutor y que, según el posicionamiento entre los modelos indicados, nos llevaría a hablar de un perfil humano y de un perfil profesional. El primero atiende a lo que es el ser y el saber estar del tutor, englobando cualidades personales deseables como:

a) Una personalidad equilibrada, sana y madura a través de la cual se desarrollen actitudes positivas y se planteen acciones objetivas que aseguren la ecuanimidad y la justicia en las decisiones (sin preferencias 0 antipatías manifiestas en el trato).

b) Una sensibilidad para captar y entender los problemas juveniles mediante la que se fomenten comportamientos empáticos y afectivos que generen un clima de acogida positivo y se visibilice el respeto hacia el estudiante acompañado de una actitud serena en las decisiones adoptadas.

c) La capacidad de entablar relaciones afectuosas y cordiales con los estudiantes desde la que acceder al conocimiento del grupo y del individuo para poder influir positivamente en sus opiniones y actitudes.

En lo que respecta al perfil profesional, se atiende al saber y al saber hacer del tutor en su dominio del conocimiento y ejercicio de la acción tutorial. En relación al conocimiento de la acción tutorial, sería deseable disponer de:

a) Un saber científico que le permita explicar de forma competente las materias que imparte.

b) Un conocimiento teórico sobre educación y ciencias afines. En concreto, es deseable conocer en profundidad las 
generalidades del sistema universitario (itinerarios, especialidades, salidas profesionales, etc.) y, a su vez, las particularidades de aquellos campos científicos que lo configuran (psicología, didáctica, filosofía, sociología, etc.).

c) Un conocimiento teórico-práctico sobre relaciones interpersonales, dirección y animación de grupos, de manera que pueda identificar estructuras, roles, factores o fuerzas que interfieren en el desarrollo del grupo de estudiantes.

d) Un conocimiento de técnicas de diagnóstico e intervención educativa de cara a una atención más personalizada, fomentar la motivación o promover iniciativas-programas de atención a la diversidad.

Por su parte, atendiendo al ejercicio práctico de la acción tutorial, también sería deseable poner en práctica técnicas ligadas a la evaluación, la realización de entrevistas, la dinamización de grupos, la promoción de habilidades sociales, la motivación, etc.

Entendemos que el perfil más idóneo de tutor universitario será aquel que conjugue un equilibrio entre las diferentes competencias creando lo que Hagenauer y Volet (2014) denominan support dimension o atmósfera de apoyo al estudiante, donde este último perciba un proceso personalizado de acompañamiento en el transcurso de su paso por la universidad y, al mismo tiempo, sienta que sus necesidades son atendidas.

\section{Necesidades tutoriales de los estudiantes universitarios}

El estudio de las necesidades de orientación que ha de atender la tutoría universitaria podría analizarse a partir de las tres fases asociadas al paso del estudiante por la universidad (ingreso, desarrollo de los estudios y la transición al mundo laboral).

Atendiendo en primer lugar a los momentos iniciales en los que este toma contacto con la enseñanza universitaria, existen numerosos estudios (Da Re y Clerici, 2017; Esteban, Bernardo y Rodríguez-Muñiz, 2016; García-Ros, Pérez-González, Pérez-Blasco y Natividad, 2012; García, Carpintero, Biencinto y Núñez, 2014; López-Gómez, 2015; Lorenzo Moledo, Argos, Hernández García y Vera Vila, 2013; Manzano-Soto y Roldán-Morales, 2015; Pujol y Durán, 2013; Rebollo y Espiñeira, 2017; Soares, Almeida y Guisande, 2011; Torrecilla et al., 2013) desde los que se podrían enfocar las demandas asociadas a tres dimensiones:

a) Lo académico (desconocimiento de la institución; desajustes competenciales ante altas exigencias; la dificultad para gestionar los tiempos de estudio; estrés ante momentos de compromiso, etc.).

b) Lo personal (falta de habilidades de autorregulación; carencias en el desarrollo de la toma de decisiones; falta de autonomía; potenciación de las estrategias metacognitivas; fortalecimiento de la motivación por los estudios, etc.).

c) Lo social (complejidad de generar nuevas redes sociales; gestión de la sepa- 
ración de los vínculos familiares; dificultades para compaginar estudios y trabajo; la potenciación de habilidades sociales y de gestión de los conflictos en el aula, etc.).

En las siguientes fases (desarrollo de los estudios y transición al mundo laboral), algunas de las necesidades comentadas pueden continuar y emerger otras nuevas (Figuera y González, 2014; Martínez Clares et al., 2019; Pérez, González, González y Martínez, 2017; Solaguren-Beascoa y Moreno, 2016):

- A nivel académico, las demandas se focalizan en dos ámbitos esenciales que tienen relación con el apoyo al proceso de enseñanza-aprendizaje (conocimiento de técnicas de estudio; orientación en los trabajos finales de estudios; conocimiento de las dinámicas de trabajo-metodologías del profesorado; mejor comprensión de los planes de estudios -asignaturas optativas, características de las materias, sistema de créditos-; dificultades ante la diversidad de sistemas de evaluación, etc.) y con el desarrollo profesional y la inserción sociolaboral (desconocimiento de las salidas profesionales; guía en la toma de decisiones ante la transición al mercado laboral; demanda de oportunidades para el desarrollo de competencias profesionales; un mayor conocimiento de la formación de posgrado, etc.).

- A nivel personal, se intensifica la necesidad de configurar una identidad consolidada (gestión de las emociones; adquisición de competencias transver- sales; apoyos en el proceso de maduración personal y en el establecimiento de metas futuras, etc.).

- A nivel social se incide en aspectos propios del desarrollo de las relaciones interpersonales (ayuda-mediación en la gestión de los conflictos; potenciación de habilidades de comunicación; asesoramiento en el desarrollo de las habilidades sociales; favorecer estrategias para trabajar en equipo, etc.).

En definitiva, entendemos que para ofrecer una óptima respuesta a estas necesidades, la acción tutorial universitaria ha de plantearse desde un posicionamiento holístico e integral pero, a su vez, adaptado a los contextos en los que se encuentra el estudiante (López y González, 2018).

\section{Planteamiento de la investiga- ción, objetivos e hipótesis}

Como objetivo general se pretende analizar el efecto que tiene el perfil y las funciones del tutor sobre las necesidades tutoriales que presenta el alumnado de educación superior; para lograrlo, se plantean los siguientes objetivos específicos:

- Validación de una escala de evaluación de la tutoría en educación superior.

- Validación del constructo de interrelación de factores asociados a la tutoría en educación superior (perfil del tutor, funciones del tutor y necesidades tutoriales).

- Confirmar la influencia positiva de los factores asociados al perfil del tutor 
sobre sus funciones y las necesidades tutoriales del alumnado en educación superior, así como la influencia de esas funciones del tutor en las necesidades tutoriales del estudiante.

GRÁFICO 1. Modelo estructural a confirmar.

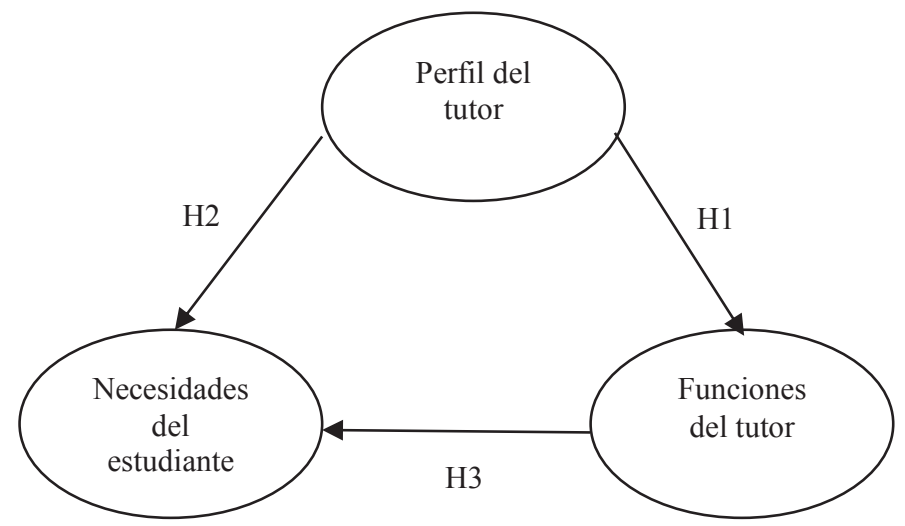

Fuente: Elaboración propia.

Siguiendo el modelo de referencia, las hipótesis que se plantean son las siguientes:

Hipótesis $1\left(\mathrm{H}_{1}\right)$ : el perfil del tutor pronostica el tipo de funciones que este ejerza (Álvarez González, 2013, 2014; Cid y Pérez, 2006; Rodríguez Espinar, 2004).

Hipótesis $2\left(\mathrm{H}_{2}\right)$ : el perfil del tutor pronostica el efecto causado por este en las necesidades tutoriales del alumnado (García Nieto et al., 2004; López et al., 2018; Martínez Clares et al., 2019).

Hipótesis $3\left(\mathrm{H}_{3}\right)$ : las funciones del tutor tienen una influencia directa sobre el efecto en las necesidades tutoriales del alumnado (Amor Almedina, 2016; López y González, 2018; Urbina et al., 2017).
Además, amparados en los fundamentos teóricos y como consecuencia de los objetivos planteados, se diseña el modelo que se somete a contraste empírico (Gráfico 1).

\section{Metodología}

Esta investigación se enmarca dentro de un diseño metodológico de tipo encuesta que pretende comprobar un modelo teórico de interrelación de diferentes factores asociados a la tutoría en educación superior, recurriendo para ello a la técnica de ecuación estructural utilizando el programa Amos 18.0.

\subsection{Participantes}

A partir de la población objeto de estudio (alumnado matriculado en titulaciones de grado de la Universidad de Huelva) se realiza un muestreo no probabilístico de tipo incidental desde el que se marcan como cuotas la representatividad de estudiantes matriculados en titulaciones pertenecientes a las diferentes áreas de conocimiento y la diversidad de género. Este procedimiento se logra 
con una muestra de 581 estudiantes $(49.1 \%$ hombres; $50.9 \%$ mujeres, con una media de edad de 22.94 años) matriculados en diversos cursos (35.5\% en primero; $27.5 \%$ en segundo; $28.1 \%$ en tercero; $9 \%$ en último curso) de treinta titulaciones diferentes [Artes y Humanidades $(4.13 \%)$, Ciencias $(3.09 \%)$, Ciencias de la Salud (8.77 \%), Ciencias Sociales y Jurídicas $(61.27 \%$ ) e Ingeniería y Arquitectura (22.71\%)]. Esta es una cifra suficiente (nivel de confianza de $95 \%$; error muestral del $5 \%$ ), aunque no óptima para una validación inicial.

\subsection{Instrumento}

Para la recogida de datos se elabora una escala ad hoc (véase Tabla 2), compuesta de tres dimensiones [Funciones del tutor (FT), Perfil del tutor (PT) y Necesi- dades tutoriales del alumnado (NT)] de 26, 13 y 21 ítems respectivamente, dispuestos en una escala tipo Likert con valores de 1 a 7 , siendo 1 nada importante y 7 muy importante. El instrumento se ha elaborado a partir de los fundamentos teóricos de referencia evidenciados en la bibliografía consultada (Álvarez González, 2013, 2014; Amor Almedina, 2016; Cid y Pérez, 2006; Echeverría, 2002; García Nieto, et al., 2004; Lázaro, 2003; López y González, 2018; Martínez Clares et al., 2019; Rodríguez Espinar, 2004, entre otros) y que dejan entrever al perfil del tutor y las funciones que desempeña, como factores asociados a una acción tutorial satisfactoria y desde los que poder llegar a conocer y atender a las necesidades tutoriales de los estudiantes universitarios.

TABLA 2. Escala de evaluación de la tutoría en educación superior.

I. Funciones del tutor (y de la tutoría) respecto de los alumnos (FT)

F1. Informar sobre cuestiones académicas institucionales (organización administrativa, plan de estudios, asignaturas, normas de promoción, itinerarios...).

F2. Proporcionar acogida institucional al alumno de nuevo ingreso.

F3. Garantizar una atención académica personalizada.

F4. Orientar y asesorar sobre estrategias de enseñanza-aprendizaje.

F5. Orientar y asesorar sobre estilos de aprendizaje, técnicas de trabajo intelectual y hábitos de estudio.

F6. Orientar para el desarrollo de planes académicos individuales.

F7. Orientar sobre el desarrollo de competencias genéricas.

F8. Realizar el seguimiento y evaluación del alumnado, proporcionándole información y asesoramiento sobre sus resultados y rendimiento.

F9. Asesorar sobre elaboración de trabajos de las asignaturas.

F10. Asesorar y recomendar bibliografía sobre las materias de su competencia.

F11. Asesorar sobre participación en la vida universitaria.

F12. Mediar en casos de conflicto (profesor/alumno; alumno/alumno; alumno/institución).

F13. Orientar sobre habilidades sociales y comunicación.

F14. Orientar sobre competencias interpersonales. 
F15. Orientar sobre resolución de conflictos en situaciones personales, familiares, de salud, sociales...).

F16. Asesorar sobre relaciones afectivas.

F17. Orientar para la toma de decisiones.

F18. Orientar sobre salidas profesionales, inserción laboral y mercado de trabajo.

F19. Asesorar para el desarrollo de competencias profesionales.

F20. Facilitar referentes profesionales.

F21. Orientar y asesorar en cuestiones sociales (vivienda, ayudas, entorno cultural...).

F22. Diagnosticar y evaluar necesidades educativas individuales y/o grupales.

F23. Coordinar o coordinarse con el equipo docente.

F24. Coordinarse con autoridades de la facultad/universidad y con el personal auxiliar (PAS, becarios...).

F25. Atender a alumnos con becas Erasmus/Sócrates.

F26. Coordinar y supervisar las prácticas de los alumnos en empresas/instituciones.

\section{Perfil humano y profesional del tutor (PT)}

P1. Afectividad.

P2. Empatía.

P3. Individualización (conocimiento personal de cada estudiante).

P4. Justicia, ecuanimidad (sin preferencias ni antipatías).

P5. Autoridad serena (capacidad de control del grupo, de hacerse respetar, no autoritarismo).

P6. Respeto a los alumnos.

P7. Personalidad capaz de influir positivamente y convencer.

P8. Capacidad de relacionarse.

P9. Capacidad de escuchar.

P10. Aptitud receptiva y disposición al diálogo.

P11. Saber de su materia (conocimiento científico).

P12. Conocimiento de las ciencias de la educación (saber sobre enseñanza).

P13. Conocimiento sobre relaciones sociales y dinámica de grupos.

\section{Necesidades tutoriales de los alumnos (NT)}

N1. Orientación en la transición de la educación secundaria a la universidad.

N2. Orientación a su llegada a la universidad (primer curso).

N3. Facilitar que el alumno pueda compartir trabajo y estudio.

N4. Orientación antes de los exámenes.

N5. Revisión de los exámenes.

N6. Orientación después de la evaluación.

N7. Orientación durante el desarrollo de las asignaturas.

N8. Consulta de dudas sobre la materia.

N9. Orientación para la mejora del rendimiento académico.

N10. Orientación por factores ligados a la edad (jóvenes, adultos, mayores...). 
N11. Orientación por cuestiones de diversidad cultural (etnia, país, grupo social...).

N12. Orientación por cuestiones de diversidad lingüística.

N13. Orientación por razones de sobredotación y altas capacidades.

N14. Orientación por necesidades educativas especiales.

N15. Orientación por discapacidad visual, auditiva, motórica...

N16. Orientación y evaluación de aptitudes (capacidades).

N17. Orientación y evaluación de actitudes y personalidad.

N18. Orientación y evaluación de estilos de aprendizaje.

N19. Orientación y evaluación de estrategias cognitivas.

N20. Orientación y evaluación de intereses profesionales.

N21. Orientación y evaluación de la motivación.

Fuente: Elaboración propia.

Para la recogida de datos, la distribución del instrumento se realizó de manera individual y con una duración de unos 15 minutos aproximadamente. Se informó a los estudiantes que participaron de forma voluntaria de las instrucciones y objetivos de la prueba, así como de la confidencialidad del procedimiento. Se aprovecharon las franjas transversales de tutorías para acceder a la población sin necesidad de interferir en los ritmos académicos. Y finalmente, para el análisis estadístico de los datos obtenidos se utilizó el software SPSS 23.

\section{Resultados}

\subsection{Validación del instrumento}

Como ya hemos mencionado, el instrumento se construye expresamente para este trabajo a partir de las referencias citadas. A continuación, se somete a juicio de expertos (seis profesores de educación de la Universidad de Huelva), lo que permite valorar tanto la pertinencia como la claridad de los ítems. Las modificaciones son menores sobre la versión inicial. Ambos procedimientos contribuyen a la validez teórica.

\subsubsection{Fiabilidad}

Aplicado el Alfa de Cronbach para estimar la consistencia interna de la escala sobre 60 variables en una muestra de 581 sujetos, se obtiene un alto índice de fiabilidad $(\alpha=.953)$ para la escala completa. En cuanto a los valores parciales de cada dimensión también nos encontramos valores altos (FT, $\alpha=.919$; PT, $\alpha=.889$; NT, $\alpha=.921)$. A continuación, se identifican aquellos ítems menos consistentes para la revisión de la escala (ítem $\mathrm{F} 6$ e ítem N1) y su mejora en futuras aplicaciones, de manera que al eliminarlos el valor total de Alfa alcanza .958 y dos de las tres dimensiones también mejoran (PT, $\alpha=.928 ; \mathrm{NT}, \alpha=.929)$.

\subsubsection{Validez de constructo}

Se realiza un Análisis Factorial Exploratorio (AFE) con la intención de concentrar la pertenencia de los ítems a un factor y así discriminar mejor entre factores. Se recurre a una reducción factorial aplican- 
do una rotación ortogonal con el método varimax. Además, atendiendo a los altos índices KMO de las escalas, con valores comprendidos entre .908 y .937 y al índice de significación $<.001$ obtenido en la prueba de esfericidad de Bartlett, se puede afirmar que el análisis factorial es pertinente, conveniente y aplicable.

A continuación, se exponen los resultados respecto a cada una de las dimensiones consideradas:

D1. Funciones del tutor respecto de los alumnos: se identifican tres factores que llegan a explicar el $53.33 \%$ de la varianza del conjunto de ítems (Tabla 3):
- Factor 1: funciones de orientación y asesoramiento general. Entre los ítems saturados por el factor se encuentran los que hacen referencia a la orientación: orientar sobre habilidades, competencias, salidas profesionales, resolución de conflictos, toma de decisiones, cuestiones sociales, estilos de aprendizaje; y los ítems que hacen referencia al asesoramiento: en competencias profesionales, relaciones afectivas, estrategias de enseñanza-aprendizaje y participación universitaria. También engloba funciones de evaluación y diagnóstico sobre necesidades educativas y funciones de coordinación de equipo docente y con autoridades (facultad/universidad).

TABLA 3. Análisis factorial sobre las funciones del tutor.

\begin{tabular}{|c|c|c|c|}
\hline \multirow{2}{*}{ Ítems } & \multicolumn{3}{|c|}{ Componentes } \\
\cline { 2 - 4 } & F1 & F2 & F3 \\
\hline F13 & .741 & -.276 & -.140 \\
\hline F14 & .722 & -.352 & -.063 \\
\hline F19 & .716 & -.200 & -.069 \\
\hline F20 & .710 & -.183 & -.075 \\
\hline F18 & .704 & -.115 & -.192 \\
\hline F7 & .692 & -.038 & .056 \\
\hline F15 & .691 & -.471 & -.050 \\
\hline F11 & .675 & -.107 & -.196 \\
\hline F16 & .669 & -.382 & .054 \\
\hline F23 & .666 & -.094 & .248 \\
\hline F2 & .653 & .049 & -.026 \\
\hline F12 & .639 & -.123 & -.175 \\
\hline F22 & .635 & -.038 & .261 \\
\hline F21 & .634 & -.256 & .049 \\
\hline F17 & .627 & -.041 & -.217 \\
\hline & & & \\
\hline
\end{tabular}


Perfiles y funciones del tutor universitario y sus efectos sobre las necesidades tutoriales del alumnado

\begin{tabular}{|c|c|c|c|}
\hline F5 & .590 & .304 & -.129 \\
\hline F24 & .578 & -.118 & .544 \\
\hline F4 & .541 & .472 & -.208 \\
\hline F1 & .514 & .436 & -.041 \\
\hline F10 & .466 & .403 & -.118 \\
\hline F9 & .384 & .667 & -.114 \\
\hline F8 & .422 & .590 & -.163 \\
\hline F3 & .480 & .523 & -.033 \\
\hline F26 & .452 & .287 & .561 \\
\hline F25 & .417 & .360 & .498 \\
\hline Varianza explicada por factor & 37.236 & 10.892 & 5.210 \\
\hline
\end{tabular}

Matriz de componentes rotados. $(\mathrm{KMO}=.917$; Bartlett. $\mathrm{Sig}=.000)$.

Fuente: Elaboración propia.

- Factor 2: funciones personalizadas en relación al seguimiento del alumnado. Este factor satura variables que ilustran a las funciones del tutor, como un asesoramiento sobre los trabajos de las asignaturas, el seguimiento y evaluación del alumnado y la atención académica personalizada.

- Factor 3: funciones relacionadas con las prácticas y becas Erasmus. Este factor satura variables que ilustran la coordinación y supervisión de las prácticas y la atención al alumnado con becas Erasmus/Sócrates.
D2. Perfil del tutor: se identifican dos factores que llegan a explicar el $56.254 \%$ de la varianza del conjunto de ítems (Tabla 4). A saber:

- Factor 1: perfil profesional. Este factor ilustra variables relacionadas con la capacidad de saber escuchar y de relacionarse, características, como la empatía, el liderazgo, la ecuanimidad o la autoridad, a la vez que muestra conocimientos sobre la educación y la materia.

TABLA 4. Análisis factorial sobre el perfil del tutor.

\begin{tabular}{|c|c|c|}
\hline \multirow{2}{*}{ Ítems } & \multicolumn{2}{|c|}{ Componentes } \\
\cline { 2 - 3 } & F1 & F2 \\
\hline P6 & .783 & .177 \\
\hline P9 & .763 & .182 \\
\hline P11 & .759 & .038 \\
\hline P12 & .736 & .030 \\
\hline
\end{tabular}


Manuel DELGADO-GARCÍA, Sara CONDE VÉLEZ y Ángel BOZA CARREÑO

\begin{tabular}{|c|c|c|}
\hline P4 & .710 & .314 \\
\hline P5 & .692 & .159 \\
\hline P7 & .641 & .242 \\
\hline P10 & .570 & .374 \\
\hline P2 & .540 & .439 \\
\hline P8 & .497 & .457 \\
\hline P1 & .051 & .882 \\
\hline P3 & .100 & .873 \\
\hline P13 & .435 & .459 \\
\hline Varianza explicada por factor & 44.591 & 11.663 \\
\hline
\end{tabular}

Matriz de componentes rotados. $(\mathrm{KMO}=.908$; Bartlett. $\mathrm{Sig}=.000)$.

Fuente: Elaboración propia.

- Factor 2: perfil humano y social. Este factor caracteriza un perfil de tutor afectivo, preocupado por conocer individualmente a cada estudiante y con conocimiento sobre relaciones sociales y dinámicas de grupos.

TABLA 5. Análisis factorial sobre las necesidades tutoriales del alumnado.

\begin{tabular}{|c|c|c|c|}
\hline \multirow{2}{*}{ Items } & \multicolumn{3}{|c|}{ Componentes } \\
\cline { 2 - 4 } & $\mathbf{1}$ & $\mathbf{2}$ & $\mathbf{3}$ \\
\hline $\mathrm{N} 18$ & .763 & .180 & .176 \\
\hline $\mathrm{N} 17$ & .733 & .371 & .113 \\
\hline $\mathrm{N} 19$ & .717 & .254 & .220 \\
\hline $\mathrm{N} 21$ & .707 & .394 & .093 \\
\hline $\mathrm{N} 20$ & .698 & .330 & .146 \\
\hline $\mathrm{N} 16$ & .615 & .465 & .133 \\
\hline $\mathrm{N} 12$ & .259 & .795 & .169 \\
\hline $\mathrm{N} 11$ & .197 & .761 & .053 \\
\hline
\end{tabular}

- Factor 1: necesidades centradas en aspectos personales $\mathrm{y} / 0$ profesionales. Este factor reúne un conjunto de ítems que enfatizan en la importancia de necesidades de orientación y evaluación sobre estilos de aprendizaje, actitudes y personalidad, estrategias cognitivas, motivación, intereses profesionales y aptitudes. can el $61.029 \%$ de la varianza del conjunto de ítems (Tabla 5). A saber: 
Perfiles y funciones del tutor universitario y sus efectos sobre las necesidades tutoriales del alumnado

\begin{tabular}{|c|c|c|c|}
\hline $\mathrm{N} 13$ & .328 & .716 & .100 \\
\hline $\mathrm{N} 10$ & .410 & .626 & .012 \\
\hline $\mathrm{N} 3$ & .312 & .585 & .279 \\
\hline $\mathrm{N} 15$ & .462 & .575 & .246 \\
\hline $\mathrm{N} 14$ & .506 & .554 & .154 \\
\hline $\mathrm{N} 2$ & .359 & .483 & .402 \\
\hline $\mathrm{N} 5$ & .037 & .129 & .802 \\
\hline $\mathrm{N} 8$ & .067 & .158 & .770 \\
\hline $\mathrm{N} 6$ & .157 & -.020 & .743 \\
\hline $\mathrm{N} 7$ & .264 & -.006 & .743 \\
\hline $\mathrm{N} 4$ & .047 & .225 & .736 \\
\hline $\mathrm{N} 9$ & .309 & .353 & .536 \\
\hline Varianza explicada por factor & 43.446 & 12.588 & 5.001 \\
\hline
\end{tabular}

Matriz de componentes rotados. $(\mathrm{KMO}=.937$; Bartlett. $\mathrm{Sig}=.000)$

Fuente: Elaboración propia.

- Factor 2: necesidades centradas en atención a la diversidad. Este factor ilustra necesidades de atención a la diversidad: lingüística, cultural, edad, discapacidades y necesidades educativas especiales.

- Factor 3: necesidades centradas en evaluación y rendimiento de asignaturas. Este factor satura una serie de necesidades cuya importancia se centra en la revisión de exámenes, dudas sobre la materia y necesidades de orientación centradas en el proceso de evaluación (antes, durante y después) con una orientación para la mejora del rendimiento académico.

\subsection{Confirmación del modelo}

Tras la reducción factorial previa se intentan contrastar empíricamente los factores extraídos. Para el modelaje de la ecuación estructural se toman como referencia las hipótesis planteadas en el modelo teórico que guía la investigación (Gráfico 1).

Los elevados índices de varianza explicados en los factores relativos a las funciones de orientación y asesoramiento general (62 \%), funciones personalizadas al seguimiento del alumnado (42\%), funciones relacionadas con las prácticas y becas erasmus (87\%), necesidades centradas en aspectos personales y/o profesionales (92\%), necesidades centradas en atención a la diversidad (87\%), necesidades centradas en evaluación y rendimiento de las asignaturas $(98 \%)$ y los índices de regresión y correlación entre los factores incluidos en la ecuación permiten la aceptación del modelo (Gráfico 2). 
GráfIco 2. Modelo de Ecuación Estructural.

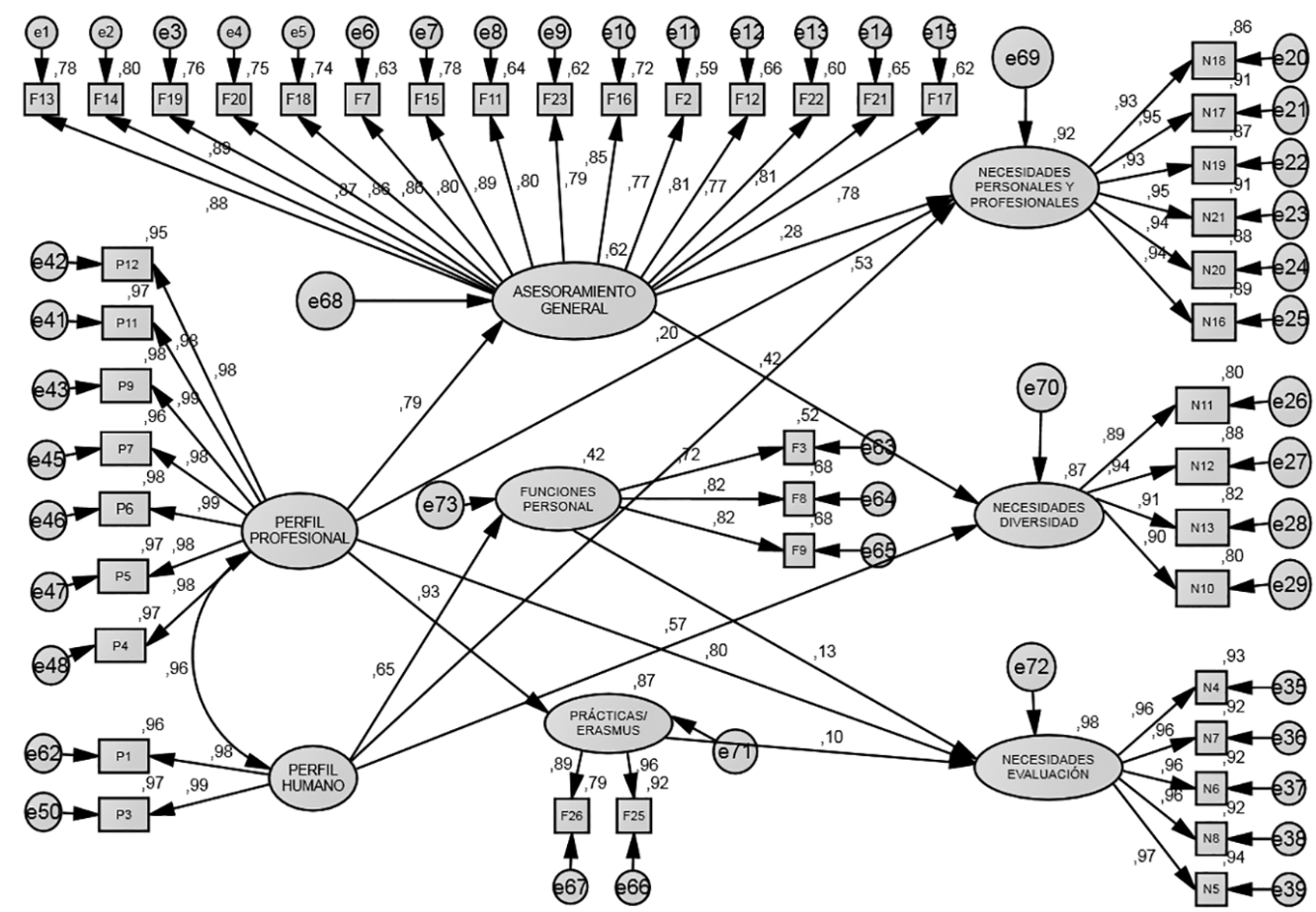

Fuente: Elaboración propia.

Los índices de bondad de ajuste (Tabla bien a los datos (Chi-square/df $=4.76 ; p>.01$; 6) indican que el modelo se ajustó bastante $\mathrm{CFI}=.93$; IFI=.93; NFI= .98; RMSA= .04).

TABLA 6. Índices de ajuste del modelo.

\begin{tabular}{|c|c|c|c|}
\hline \multicolumn{2}{|r|}{ Índices de ajuste } & $\begin{array}{c}\text { Valores } \\
\text { recomendados }\end{array}$ & $\begin{array}{c}\text { Valores } \\
\text { observados }\end{array}$ \\
\hline $\begin{array}{l}\text { Índice de ajuste } \\
\text { absoluto }\end{array}$ & $\mathrm{X}^{2} / \mathrm{gl}$ (Chi-cuadrado/gl) & $\leq 5.00$ & $4.76 ; \mathrm{p}<.001$ \\
\hline \multirow{3}{*}{$\begin{array}{l}\text { Índice de ajuste } \\
\text { comparativo }\end{array}$} & IFI(índice de ajuste incremental) & $\geq .90$ & .93 \\
\hline & NFI(índice de ajuste normalizado) & $\geq .90$ & .98 \\
\hline & CFI(índice de ajuste comparativo) & $\geq .90$ & .93 \\
\hline $\begin{array}{l}\text { Error de } \\
\text { aproximación }\end{array}$ & $\begin{array}{c}\text { RMSEA(raíz cuadrada del error } \\
\text { medio cuadrático) }\end{array}$ & $\leq .06$ & .04 \\
\hline Ajuste de muestra & HOELTER .05 & $>200$ & 480 \\
\hline
\end{tabular}

Fuente: Elaboración propia. 
En el modelo se observan tres grupos de elementos, por una parte, los factores independientes que hacen referencia al perfil del tutor (social y profesional) y, por otra parte, como variables dependientes, las funciones que realizan los tutores, medidas en tres tipos (de orientación y asesoramiento general, personalizadas y relacionadas con las prácticas y becas Erasmus/Sócrates) y, por último, las necesidades tutoriales del alumnado (sobre aspectos personales $\mathrm{y} / 0$ profesionales; centradas en atención a la diversidad; relativas a la evaluación y rendimiento en asignaturas).

El modelo plantea correlaciones entre el perfil del tutor y regresiones o influencias de estos perfiles sobre las funciones y las necesidades. De ahí que, comenzando con las correlaciones entre los factores independientes, se identifique un elevado nivel de correlación entre el perfil humano y social y el perfil profesional $(r=.96$; $\mathrm{p} \leq .01) \mathrm{y}$, profundizando en el análisis de los índices de regresión entre factores, se encuentren los siguientes resultados:

a) En función del tipo de perfil asociado al tutor se puede pronosticar el tipo de funciones que este ejerce, así como las necesidades tutoriales del alumnado a las que atiende; en este sentido, se observa en el modelo cómo un perfil de tutor más profesional muestra una influencia positiva y significativa sobre las funciones de orientación, asesoramiento general $(\beta=.79 ; p \leq .01)$ y prácticas Erasmus $(\beta=.93 ; p \leq .01)$ y responde a un tipo de necesidades centradas tanto en aspectos persona- les/profesionales $(\beta=.20 ; \mathrm{p} \leq .01)$ como en necesidades de evaluación y rendimiento del alumnado $(\beta=.80 ; p \leq .01)$. También se observa cómo un perfil más humano/social pronostica funciones más personalizadas orientadas al seguimiento del alumnado $(\beta=.65 ; p \leq .01)$, atendiendo a necesidades personales, profesionales $(\beta=.53 ; p \leq .01)$ y de di$\operatorname{versidad}(\beta=.57 ; \mathrm{p} \leq .01)$.

b) Respecto a las funciones, se observa cómo, dependiendo de la tipología que se desarrolle, influyen positivamente y en distinto nivel sobre las necesidades tutoriales, siendo estas igualmente determinadas por el perfil que defina al tutor. En este sentido, se aprecia cómo las funciones de orientación y asesoramiento general influyen positiva y significativamente sobre las necesidades centradas en aspectos personales y/0 profesionales $(\beta=.28 ; p \leq .01)$, y sobre las ligadas a la atención a la diversidad $(\beta=.42 ; p \leq .01)$.

c) Finalmente, las necesidades centradas en la evaluación y seguimiento de las asignaturas vienen pronosticadas por funciones personalizadas al seguimiento del alumnado $(\beta=.13 ; \mathrm{p} \leq$ $.01) \mathrm{y}$, en menor nivel, por funciones relacionadas con las prácticas y becas Erasmus $(\beta=.10 ; \mathrm{p} \leq .01)$.

Finalmente, respecto al modelo de medida, para aquellos factores que saturan a una gran cantidad de indicadores, se decide incluir los ítems cuyas cargas factoriales son superiores a 6 ya que se consigue un mejor ajuste (Tabla 7): 
Manuel DELGADO-GARCÍA, Sara CONDE VÉLEZ y Ángel BOZA CARREÑo

TABLA 7. Cargas factoriales del modelo de medida.

\begin{tabular}{|c|c|}
\hline Indicador & Valores recomendados \\
\hline \multicolumn{2}{|l|}{ ASESORAMIENTO GENERAL } \\
\hline F13 & .885 \\
\hline F14 & .893 \\
\hline F19 & .870 \\
\hline F20 & .863 \\
\hline F18 & .858 \\
\hline F7 & .796 \\
\hline F15 & .886 \\
\hline F11 & .801 \\
\hline F23 & .785 \\
\hline F16 & .851 \\
\hline F2 & .768 \\
\hline F12 & .813 \\
\hline F22 & .772 \\
\hline F21 & .806 \\
\hline F17 & .785 \\
\hline \multicolumn{2}{|c|}{ NECESIDADES PERSONALES Y PROFESIONALES } \\
\hline N18 & .926 \\
\hline N17 & .955 \\
\hline N19 & .935 \\
\hline $\mathrm{N} 21$ & .952 \\
\hline $\mathrm{N} 20$ & .937 \\
\hline $\mathrm{N} 16$ & .942 \\
\hline \multicolumn{2}{|l|}{ NECESIDADES DIVERSIDAD } \\
\hline N11 & .893 \\
\hline $\mathrm{N} 12$ & .938 \\
\hline $\mathrm{N} 13$ & .906 \\
\hline $\mathrm{N} 10$ & .896 \\
\hline \multicolumn{2}{|l|}{ NECESIDADES EVALUACIÓN } \\
\hline N4 & .965 \\
\hline N7 & .959 \\
\hline N6 & .957 \\
\hline
\end{tabular}


Perfiles y funciones del tutor universitario y sus efectos sobre las necesidades tutoriales del alumnado

\begin{tabular}{|c|c|}
\hline N8 & .961 \\
\hline N5 & .972 \\
\hline \multicolumn{2}{|l|}{ PERFIL PROFESIONAL } \\
\hline $\mathrm{P} 11$ & .983 \\
\hline $\mathrm{P} 12$ & .977 \\
\hline P9 & .987 \\
\hline $\mathrm{P} 7$ & .979 \\
\hline $\mathrm{P} 6$ & .990 \\
\hline P5 & .983 \\
\hline $\mathrm{P} 4$ & .985 \\
\hline \multicolumn{2}{|l|}{ PERFIL HUMANO } \\
\hline P3 & .987 \\
\hline $\mathrm{P} 1$ & .980 \\
\hline \multicolumn{2}{|l|}{ FUNCIONES PERSONAL } \\
\hline F3 & .720 \\
\hline F8 & .824 \\
\hline F9 & .824 \\
\hline \multicolumn{2}{|l|}{ PRÁCTICAS ERASMUS } \\
\hline F26 & .888 \\
\hline F25 & .960 \\
\hline
\end{tabular}

Fuente: Elaboración propia.

\section{Discusión y conclusiones}

El objetivo de este trabajo era analizar el grado de relación que tienen el perfil y las funciones del tutor sobre las necesidades tutoriales que presenta el alumnado de educación superior. Para ello, se establecieron dos objetivos específicos que acotaran la validación de un instrumento y del constructo objeto de estudio para lograr un análisis válido y fiable de los resultados obtenidos, lo cual se ha visto confirmado a través del AFE y la posterior confirmación de un modelo, mediante el procedimiento de ecuaciones estructurales, que nos lleva a identi- ficar la existencia de correlaciones entre el perfil del tutor, y regresiones o influencias de estos perfiles sobre sus propias funciones y las necesidades de los estudiantes.

Concretando los objetivos y atendiendo a las hipótesis planteadas, los resultados nos permiten confirmar dichas hipótesis a través de diferentes correlaciones evidenciadas. Como conclusiones destacan:

a) Existe un perfil profesional de docente universitario que otorga prioridad al área académica de la acción tutorial con 
el fin de atender a unas necesidades de los estudiantes ligadas a la evaluación y al rendimiento académico; esto le exige demostrar ciertas competencias profesionales (saber escuchar y saber relacionarse y demostrar conocimientos sobre la educación y la materia que imparte) y cualidades en su personalidad (empatía, liderazgo, ecuanimidad 0 autoridad) que son compartidas por otras investigaciones (Fernández-Salinero, 2014; García Cabrero, et al., 2016; Martínez Clares et al., 2014; Más Torelló, 2012; Torrecilla et al., 2013). Este perfil de tutor también se relaciona con el desarrollo de funciones personalizadas y próximas al seguimiento del alumnado (asesorar sobre los trabajos de las asignaturas, el seguimiento y evaluación del alumnado y la atención académica personalizada) y con funciones ligadas al asesoramiento en los períodos de prácticas y/o en pro-

b) Existe un perfil humano de docente universitario que desarrolla más intensamente el área personal y social de la acción tutorial, con el fin de atender a unas necesidades de los estudiantes próximas a aspectos íntimos de su personalidad, la toma de decisiones profesionales o la atención a la diversidad; esto le exige demostrar un comportamiento afectivo, una preocupación por la personalización e individualización de la enseñanza e implicarse en las relaciones sociales y dinámicas de los grupos. Este perfil de tutor desarrolla unas funciones orientadoras y de asesoramiento general (orientar sobre habilidades, competencias, salidas profesionales, resolución de conflictos, toma de decisiones, cuestiones sociales, estilos de aprendizaje, asesoramiento en competencias profesionales, relaciones afectivas, estrategias de enseñanza-aprendizaje y participación universitaria) que, aunque no cuentan con la trayectoria de contextos como el anglosajón (Cashmore, Scott y Cane, 2012, analizaban la importancia del rol del tutor para generar un sentimiento de pertenencia con la institución y evitar el abandono temprano), en la actualidad emergen como una de las funciones claves en la tutoría universitaria y de ahí que haya que profundizar en los principios que han de guiar su desarrollo (Haya, Calvo y Rodríguez, 2013; Herrera Rodríguez, 2017; López y González, 2018; López et al., 2013; Manzano-Soto y Roldán-Morales, 2015; Martínez Clares et al., 2019; Yale, 2019).

Se hacen visibles dos modelos de acción tutorial (académico-profesional - teaching-y personal —counseling-) delimitados por las necesidades que presenta el alumnado y que polarizan la labor y el perfil del tutor universitario, por lo que esta contribución apoya las tesis teóricas existentes desde las que se configuran las diferentes clasificaciones de modelos de tutoría universitaria (Álvarez González, 2013, 2014; Álvarez González y Álvarez, 2015; Lobato y Guerra, 2016) y pone de relieve la necesidad de tomar en consideración las necesidades del estudiante como punto de partida desde el que diseñar y desarrollar aquellas prácticas tutoriales incluidas en los diferentes servicios y/o programas que se desarrollan en la universidad. 
Para alcanzar este reto, el estudio que se presenta comparte con otros trabajos recientes (Martínez Clares et al., 2019; Waltz, 2019) la importancia de proporcionar al profesorado información y formación práctica para generar una «concienciación profesional» desde la que adquirir un compromiso con una función inherente a la labor docente y que «no ha de basarse únicamente en aspectos técnicos y metodológicos, sino también en aquellas competencias personales y participativas que la tutoría reclama» (Martínez Clares et al., 2016, p. 95). Frente a contextos masificados y proclives a la despersonalización del proceso de enseñanza y aprendizaje, los estudiantes expresan claramente una preocupación en este sentido y trabajos como el de Amor Almedina (2016), Cashmore et al. (2012), López-Gómez (2017) o Pérez et al. (2017) demuestran que aquellos tutores más cercanos al estudiante generan en ellos un mejor proceso de adaptación a la universidad, desarrollan un sentimiento de pertenecía a la institución e incrementan sus expectativas de éxito y rendimiento académico.

En definitiva, la propuesta de una acción tutorial integral (Álvarez González, 2017) ha de tener cabida en la labor del tutor universitario y así queda demostrado en gran parte de la teoría que sirve de fundamentación del presente estudio y en los resultados obtenidos en el mismo.

\section{Limitaciones y líneas de inves- tigación}

Como limitaciones del estudio cabría señalar la necesidad de contrastar la opinión del alumnado con el tutor universitario, de manera que se logre un mayor ajuste y significación de los objetivos propuestos. Del mismo modo, haber incrementado la muestra final en algunas de las áreas de conocimiento hubiese ofrecido una mayor generalización de una información que parte de una realidad concreta, pero que se asemeja a otros contextos analizados en la fundamentación del estudio.

Como prospectiva, este trabajo promueve la necesidad de continuar indagando en las necesidades del alumnado universitario y en la labor del docente como tutor para generar un contexto de enseñanza y aprendizaje significativo para ambos agentes. A ello puede contribuirse mediante la construcción y validación de instrumentos que atiendan a constructos y variables asociadas a la acción tutorial en la educación superior, así como la transferencia de información procedente de otros contextos internacionales con trayectorias dilatadas en el desarrollo de la tutoría universitaria.

\section{Referencias bibliográficas}

Álvarez González, M. (2013). Hacia un modelo integral de la tutoría universitaria. En M. Álvarez González y R. Bisquerra (Coords.), Manual de orientación y tutoría [CD]. Barcelona: Wolters Kluwer España.

Álvarez González, M. (2014). La orientación y la tutoría universitaria en la formación de los grados y postgrados. Las transiciones académicas. En P. Figuera (Coord.), Persistir con éxito en la Universidad: De la investigación a la acción (pp.175-203). Barcelona: Laertes.

Álvarez González, M. (2017). Hacia un modelo integrador de la tutoría en los diferentes niveles educativos. Educatio Siglo XXI, 35 (2), 21-42. 
Álvarez González, M. y Álvarez, J. (2015). La tutoría universitaria: del modelo actual a un modelo integral. Revista Electrónica Interuniversitaria de Formación del Profesorado, 18 (2), 125-142. doi: http://dx.doi.org/10.6018/reifop.18.2.219671

Álvarez González, M. y Bisquerra, R. (2012). Orientación educativa. Modelos, áreas estrategias y recursos. Madrid: Wolters Kluwer España.

Álvarez-Pérez, P. R. (2014). La función tutorial del profesorado universitario: Una nueva competencia de la labor docente en el contexto del EEES. Revista Portuguesa de Pedagogía, 47 (2), 85-106

Álvarez-Pérez, P. R., López, D. y Pérez, D. (2016). Programa de tutoría universitaria formativa y desarrollo del proyecto personal del alumnado. Revista de Pedagogía, 37 (100), 67-89.

Amor Almedina, M. (2016). Evaluación de la orientación y la tutoría en la Facultad de Ciencias de la Educación de la Universidad de Córdoba. Educatio Siglo XXI, 34 (1), 93-11.

Arza, N., Salvador, X. y Mascarenhas, S. (2014). La función tutorial del profesorado: Estudio evaluativo en tres universidades federales brasileñas. Revista Electrónica Interuniversitaria Educativa, 20 (1), 7-43.
Esteban, M., Bernardo, A. B. y Rodríguez-Muñiz, L. J. (2016). Permanencia en la universidad: la importancia de un buen comienzo. Aula Abierta, 44, 1-6. doi: https://doi.org/10.1016/j. aula.2015.04.001

Fernández-Salinero, C. (2014). La tutoría universitaria en el EEES: Perfiles actuales. Teoría de la Educación, 26, 161-186. doi: http:/dx.doi. org/10.14201/teoredu2014261161186

Figuera, P. y González, M. Á. (2014). La intervención orientadora y tutorial en la adaptación y persistencia del alumnado en la universidad. Revista de orientación educacional, 28 (54), 31-50.

Gaitán, P. (2013). Hacia una definición de tutoría universitaria. Didac, 14 (61), 4-8. Recuperado de https://bit.ly/31ES4rD (Consultado el 24-112018).

García Cabrero, B., Ponce Ceballos, S., García Vigil, M. H., Caso Niebla, J., Morales Garduño, C., Martínez Soto, Y., ... Aceves Villanueva, Y. (2016). Las competencias del tutor universitario: una aproximación a su definición desde la perspectiva teórica y de la experiencia de sus actores. Perfiles educativos, 38 (151), 104-122.

García Nieto, N. (2011). La función tutorial en el ámbito educativo. Padres y Maestros, 342, 5-9.

García Nieto, N., Asensio Muñoz, I., Carballo Santaolalla, R., García García, M. y Guardia González, S. (2004). Guía para la labor tutorial en la Universidad en el Espacio Europeo de Educación Superior. Madrid: MECD.

García, M., Carpintero, E., Biencinto, C. y Núñez, M. C. (2014). La evaluación del proyecto SOU-estuTUtor: percepción de los mentores como punto de referencia para la mejora de un programa de mentoría universitaria para estudiantes de primero. Revista Complutense de Educación, 25 (2), 433-455.

García-Ros, R., Pérez-González, F., Pérez-Blasco, J. y Natividad, L. (2012). Evaluación del estrés académico en estudiantes de nueva incorporación a la universidad. Revista Latinoamericana de Psicología, 44 (2), 143-154. Recuperado de https://bit.ly/2BwzOq7 (Consultado el 20-122018). 
García-Valcárcel, A. (2008). La tutoría en la enseñanza universitaria y la contribución de las TIC para su mejora. RELIEVE, 14 (2), 1-14. Recuperado de https://bit.ly/2JakwLI

Giner, A. (2012). Perfil competencial del tutor o de la tutora de enseñanza secundaria. Revista Española de Orientación y Psicopedagogía, 23 (2), 22-41. doi: https://doi.org/10.5944/reop.vol.23. num.2.2012.11416

González-Benito, A. y Vélaz de Medrano, C. (2014). La acción tutorial en el sistema escolar. Madrid: UNED.

González-Lorente, C. y González-Morga, N. (2015). Enseñar a transitar desde la Educación Primaria: el proyecto profesional y vital. Revista Electrónica Interuniversitaria de Formación del profesorado, 18 (2), 29-41. doi: https://doi. org/10.6018/reifop.18.2.219291

Hagenauer, G. y Volet, S. E. (2014). Teacher-student relationship at university: an important yet under-researched field. Oxford Review of Education, 40 (3), 370-388.

Haya, I., Calvo, A. y Rodríguez, C. (2013). La dimensión personal de la tutoría universitaria: Una investigación cualitativa en la Universidad de Cantabria (España). Revista Española de Orientación y Psicopedagogía, 24 (3), 98113. doi: https://doi.org/10.5944/reop.vol.24. num.3.2013.11247

Herrera Rodríguez, M. G. (2017). Las tutorías, un apoyo trascendente para los universitarios. Revista Iberoamericana de Producción Académica y Gestión Educativa, 7 (4), 1-12. Recuperado de https://bit.ly/2Pf88hc (Consultado el 24-112018).

Lázaro, A. (2003). Competencias tutoriales en la universidad. En F. Michavilla y J. García (Eds.), La tutoría y los nuevos modos de aprendizaje en la universidad (pp. 107-128). Madrid: CAM-Cátedra UNESCO.

León, V. (2018). Funcionamiento de la acción tutorial y factores de eficacia (Tesis doctoral inédita). Universidad Complutense de Madrid, Madrid.

Lobato, C. y Guerra, N. (2016). La tutoría en la educación superior en Iberoamérica: Avances y desafíos. Revista Educar, 52 (2), 379-398. doi: https://doi.org/10.5565/rev/educar.726
López-Gómez, E. (2015). La tutoría en el EEES: propuesta, validación y valoración de un modelo integral (Tesis Doctoral). Repositorio Institucional de la Universidad Nacional de Educación a Distancia, Madrid. Recuperado de https://bit.ly/2JbCPAe

López-Gómez, E. (2017). El concepto y las finalidades de la tutoría universitaria. Una consulta a expertos. Revista Española de Orientación y Psicopedagogía, 28 (2), 61-78. doi: https://doi. org/10.5944/reop.vol.28.num.2.2017.20119

López, I. y González, P. (2018). La tutoría universitaria como espacio de relación personal. Un estudio de caso múltiple. Revista de Investigación Educativa, 36 (2), 381-399. doi: http://dx.doi. org/10.6018/rie.36.2.291161

López, I., González, P. y Velasco, P. J. (2013). Ser y Ejercer de tutor en la universidad. Revista de Docencia Universitaria, 11 (2), 107-134.

Lorenzo Moledo, M., Argos, J., Hernández García, J. y Vera Vila, J. (2013). El acceso y la entrada del estudiante a la universidad: situación y propuestas de mejora facilitadoras del tránsito. Educación XX1, 17 (1), 15-38.

Manzano-Soto, N. y Roldán-Morales, C. (2015). Análisis de necesidades de orientación del estudiante de primer año en la universidad autónoma de occidente. Revista Española de Orientación y Psicopedagogía, 26 (3), 121140. doi: https://doi.org/10.5944/reop.vol.26. num.3.2015.16404

Martínez-Mínguez, L. y Moya, L. (2017). La opinión de los estudiantes: la tutoría académica en la adquisición de competencias profesionales de educación física en los graduados en educación infantil. DIDACTICAE, 2, 71-88.

Martínez Clares, P., Martínez Juárez, M. y Pérez Cusó, J. (2014). Tutoría Universitaria: entorno emergente en la Universidad Europea. Un estudio en la Facultad de Educación de la Universidad de Murcia. Revista de Investigación Educativa, 32 (1), 111-138.

Martínez Clares, P., Martínez Juárez, M. y Pérez Cusó, J. (2016). ¿Cómo avanzar en la tutoría universitaria? Estrategias de acción: los estudiantes tienen la palabra. Revista Española de Orientación y Psicopedagogía, 27 (2), 8098. doi: https://doi.org/10.5944/reop.vol.27. num.2.2016.17115 
Martínez Clares, P., Pérez Cusó, F. J. y González Morga, N. (2019). ¿Qué necesita el alumnado de la tutoría universitaria? Validación de un instrumento de medida a través de un análisis multivariante. Educación XX1, 22 (1), 189-213. doi: https://doi.org/10.5944/educXX1.21302

Más Torelló, Ó. (2012). Las competencias del docente universitario: la percepción del alumno, de los expertos y del propio protagonista. Revista de Docencia Universitaria, 10 (2), 299318.

McFarlane, K. J. (2016). Tutoring the tutors: supporting effective personal tutoring. Active Learning in Higher Education, 17 (1), 77-88. doi: https://doi.org/10.1177/1469787415616720

Pantoja, A. (2013). La acción tutorial en la escuela. Madrid: Editorial Síntesis.

Pérez, F. J., González, C., González, N. y Martínez, M. (2017). Tutoría en la Universidad: un estudio de caso en la Facultad de Educación de la Universidad de Murcia. Educatio Siglo XXI, 2 (35), 91-110.

Pujol, E. y Durán, E. (2013). Manejo del tiempo académico en jóvenes que inician estudios en la Universidad Simón Bolívar. Latinoamericana de Ciencia Sociales, Niñez y Juventud, 11 (1), 93-108.

Real Decreto 1791/2010, de 30 de diciembre, por el que se aprueba el Estatuto del Estudiante Universitario. Boletín Oficial del Estado, 318, de 31 de diciembre de 2010, páginas 109353 a 109380. Recuperado de https://www.boe.es/boe/ dias/2010/12/31/pdfs/BOE-A-2010-20147.pdf (Consultado el 22-10-2019).

Rebollo, N. y Espiñeira, E. M. (2017). La tutoría durante el proceso de desarrollo del TFG y TFM: análisis del grado de utilidad y satisfacción del alumnado. Educatio Siglo XXI, 35 (2), 161-180.

Rodríguez Espinar, S. (2004). Manual de tutoría universitaria. Recursos para la acción. Barcelona: Octaedro-ICE.

Soares, A. P. C., Almeida, L. S. y Guisande, M. A. (2011). Ambiente académico y adaptación a la universidad: un estudio con estudian-

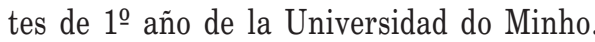
Revista iberoamericana de psicología y salud, 2 (1), 99-121. Recuperado de https://bit. $\mathrm{ly} / 33 \mathrm{~W} 2 \mathrm{oNt}$
Solaguren-Beascoa, M. y Moreno, L. (2016). Escala de actitudes de los estudiantes universitarios hacia las tutorías académicas. Educación XX1, 19 (1), 247-266. doi: https://doi. org/10.5944/educxx1.15586

Torrecilla, E. M., Rodríguez, M. J., Herrera, M. E. y Martín, J. F. (2013). Evaluación de calidad de un proceso de tutoría de titulación universitaria: La perspectiva del estudiante de nuevo ingreso en educación. Revista Española de Orientación y Psicopedagogía, 24 (2), 79-99. doi: https://doi.org/10.5944/reop.vol.24. num.2.2013.11260

Troyano, Y. y García, A. J. (2009). Expectativas del alumnado sobre el profesorado tutor en el contexto del Espacio Europeo de Educación Superior. Revista Docencia Universitaria, 3, 1-8. Recuperado de https://bit.ly/2J74XEH

Urbina, A., de la Calleja, J. y Medina, M. A. (2017). Associating students and teachers for tutoring in higher education using clustering and data mining. Computers Applications in Engineering Education, 25 (5), 823-832.

Waltz, S. B. (2019). Tutor training for service learning: impact on self-efficacy beliefs. Mentoring \& Tutoring: Partnership in Learning, 27 (1), 26-43. doi: https://doi.org/10.1080/1361 1267.2019.1583405

Yale, A. T. (2019). The personal tutor-student relationship: student expectations and experiences of personal tutoring in higher education. Journal of Further and Higher Education, 43 (4), 533-544. doi: https://doi.org/10.1080/0309877X.2017.1377164 


\section{Biografía de los autores}

Manuel Delgado-García es Licenciado y Doctor en Psicopedagogía por la Universidad de Huelva y Licenciado en Pedagogía por la Universidad Nacional de Educación a Distancia (UNED). Profesor Ayudante Doctor en el Departamento de Pedagogía de la Universidad de Huelva y colaborador con Universidad Internacional de la Rioja (UNIR). Sus líneas de investigación giran en torno la orientación, la acción tutorial y el desarrollo profesional docente.

(iD https://orcid.org/0000-0001-6157-0494

Sara Conde Vélez es Licenciada y Doctora en Psicopedagogía por la Universidad de Huelva. Profesora Ayudante Doctora en el Departamento de Pedagogía de la Universidad de Huelva. Sus principales líneas de investigación giran en torno a la convivencia escolar, el acoso escolar y diseños y validación de instrumentos de evaluación.

(iD https://orcid.org/0000-0002-7950-5866
Ángel Boza Carreño es Licenciado en Pedagogía por la UNED y Doctor en Psicopedagogía por la Universidad de Huelva. Orientador educativo (1983-1997) y Profesor Titular del Área de Métodos de Investigación y Diagnóstico en Educación, integrada en el Departamento de Pedagogía de la Universidad de Huelva. Sus líneas de investigación giran en torno la orientación educativa y la acción tutorial, así como a los métodos de investigación en educación.

iD https://orcid.org/0000-0002-3395-421X 


\section{revista española de pedagogía} año 78, n² 275, enero-abril 2020

Spanish Journal of Pedagogy

year 78, n. 275, January-April 2020

\section{Sumario*}

\section{Table of Contents **}

\section{Cuestiones de enseñanza de las matemáticas}

Wendolyn Elizabeth Aguilar-Salinas, Maximiliano de las Fuentes-Lara, Araceli Celina Justo-López y Ana Dolores Martínez-Molina

Instrumento de medición para diagnosticar las habilidades algebraicas de los estudiantes en el Curso de Cálculo Diferencial en ingeniería

A measurement instrument for establishing the algebraic skills of engineering students on a Differential Calculus Course in engineering

Maria Burgos, Pablo Beltrán-Pellicer y Juan D. Godino La cuestión de la idoneidad de los vídeos educativos de matemáticas: una experiencia de análisis con futuros maestros de educación primaria

The issue of didactical suitability in mathematics educational videos: experience of analysis with prospective primary school teachers

\section{La formación online en el mundo de la educación: experiencias de los Estados Unidos}

Gerald LeTendre y Tiffany Squires

Integración de programas de máster online y presenciales en educación

Integrating online and residential master's programs in education

\section{Laurence B. Boggess}

Innovación en la capacitación docente online: un modelo organizacional para brindar apoyo a largo plazo a la docencia online Innovations in online faculty development: an organizational model for long-term support of online faculty

Brian Redmond

Liderazgo de equipo compartido de un programa online

27 Shared team leadership for an online program

\footnotetext{
* Todos los artículos están también publicados en inglés en la página web de la revista: https://revistadepedagogia.org.

** All the articles are also published in English on the web page of the journal: https://revistadepedagogia.org.
} 


\section{Estudios y Notas}

\section{Paolo Scotton}

Pensar en común, vivir en plenitud. La experiencia de

la filosofía con los niños y niñas

Thinking together, living fully. Experiencing philosophy with

children

103

\section{Manuel Delgado-Garcia, Sara Conde Vélez y Ángel}

Boza Carreño

Perfiles y funciones del tutor universitario y sus

efectos sobre las necesidades tutoriales del alumnado

Profiles and functions of university tutors and their effects on

students' tutorial needs

119

\section{Reseñas bibliográficas}

Luri, G. (2019). La imaginación conservadora: una defensa apasionada de las ideas que han hecho del mundo un lugar mejor (Enrique Alonso Sainz).

Quigley, C. F. y Herro, D. (2019). An educator's guide to STEAM. Engaging students using real-world problems (Juan Luis Fuentes).Prince, T. (2019). Ejercicios de mindfulness en el aula. 100 ideas prácticas (José V. Merino Fernández).

\section{Informaciones}

Francisco Altarejos: la filosofía de la educación hecha vida (Concepción Naval); World Educational Research Association Focal Meeting 2020: «Networking Education: Diverse Realities, Common Horizons»);

Conclusiones del IX Congreso Internacional de Filosofía de la Educación; Ebook 2019: José Antonio Ibáñez-Martín (Ed.) (2019). La misión de las revistas de investigación en el mundo educativo. The mission of research journals in the world of education.

\section{Instrucciones para los autores}

Instructions for authors

\section{Solicitud de originales}

Call for papers

169

XV Congreso Internacional de Teoría de la Educación

XV International Congress on Theory of Education

ISSN: 0034-9461 (Impreso), 2174-0909 (Online)

https://revistadepedagogia.org/

Depósito legal: M. 6.020 - 1958

INDUSTRIA GRÁFICA ANZOS, S.L. Fuenlabrada - Madrid 


\section{Profiles and functions of university tutors and their effects on students' tutorial needs}

\section{Perfiles y funciones del tutor universitario y sus efectos sobre las necesidades tutoriales del alumnado}

Manuel DELGADO-GARCÍA, PhD. Assistant Professor. Universidad de Huelva (manuel.delgado@dedu.uhu.es). Sara CONDE VÉLEZ, PhD. Assistant Professor. Universidad de Huelva (sara.conde@dedu.uhu.es). Ángel BOZA CARREÑo, PhD. Associate Professor. Universidad de Huelva (aboza@uhu.es).

\section{Abstract:}

Large numbers of students on particular degrees and a progressive depersonalisation of the teaching and learning process are typical characteristics of higher education. This situation results in a need to analyse the tutorial activity processes currently in use. This work aims to investigate various factors associated with the main agents involved in this process: tutors (profiles and functions) and students (needs). It is based on a survey-type methodology creating an instrument for distribution among degree students at the Universidad de Huelva. This is first subjected to validation processes to obtain a theoretical reference model with which to evaluate interactions among the emerging factors.

Notable results include a theoretical model with correlations between tutor profiles and regressions, or influences of these profiles on functions and student needs. Furthermore, it reveals two types of university tutor: those who prioritise the academic aspects of tutoring, and those who prioritise personal aspects. Both profiles are associated with

Revision accepted: 2019-09-18.

This is the English version of an article originally printed in Spanish in issue 275 of the revista española de pedagogía. For this reason, the abbreviation EV has been added to the page numbers. Please, cite this article as follows: Delgado-García, M., Conde Vélez, S., \& Boza Carreño, Á. (2020). Perfiles y funciones del tutor universitario y sus efectos sobre las necesidades tutoriales del alumnado | Profiles and functions of university tutors and their effects on students' tutorial needs. Revista Española de Pedagogía, 78 (275), 119-143. doi: https://doi.org/10.22550/REP78-12020-03 
types of function that react to the needs students display throughout their time at university, and support the thesis that university tutoring is developing towards an integral model in which the personal dimension is especially relevant.

Keywords: educational guidance, tutoring, higher education, student guidance needs, competence profile of tutors.

\section{Resumen:}

La educación superior se caracteriza por una masificación de estudiantes en determinadas titulaciones y una progresiva despersonalización del proceso de enseñanza y aprendizaje. Esta realidad justifica la necesidad de analizar los procesos de acción tutorial que se desarrollan en la actualidad. Este trabajo tiene como objetivo indagar en varios factores asociados a los principales agentes implicados en dicho proceso: tutor (perfiles y funciones) y alumnado (necesidades). Se parte de una metodología tipo encuesta en la que se construye y se somete a procesos de validación un instrumento distribuido entre los estudiantes de grado de la Universidad de Huelva, con el objetivo de obtener un modelo teórico de referencia desde el que valorar las interacciones entre los factores emergentes.

Como resultados, cabe destacar un modelo teórico en el que existen correlaciones entre el perfil del tutor, y regresiones o influencias de estos perfiles sobre las funciones y las necesidades de los estudiantes; de la misma manera, se visibilizan dos tipologías de tutor universitario que priorizan los aspectos académicos y personales de la tutoría respectivamente. Ambos perfiles llevan asociados unos tipos de funciones que vendrán a dar respuesta a las necesidades que presentan los estudiantes a lo largo de su paso por la universidad y que apoyan las tesis del avance de la tutoría universitaria hacia un modelo integral en el que la dimensión personal cobra una especial relevancia.

Descriptores: orientación educativa, tutoría, educación superior, necesidades de orientación del alumnado, perfil competencial del tutor.

\section{Introduction}

Tutoring in higher education is regarded as a function that forms an integral part of the teacher's role. It is a personalised model for education that provides comprehensive support for students and helps to «reduce anxiety, encourage integration in the new institution, and improve conditions for learning» (Fernández-Salinero, 2014, p. 163). As well as these factors, there are other elements associated with $21^{\text {st }}$ century university (a 
diverse student body, curriculum diversification, uncertainty about the future and finding work, and constant social, educational, and professional changes) that lead to a need to integrate tutoring into the curriculum and for institutional policies to promote it (Álvarez González, 2017; Martínez Clares, Pérez Cusó, \& González Morga, 2019).

Several definitions are currently used for tutoring action or tutoring (Álvarez González \& Álvarez, 2015; Álvarez González \& Bisquerra, 2012; Álvarez-Pérez, 2014; González-Benito \& Vélaz de Medrano, 2014; Hagenauer \& Volet, 2014; Pantoja, 2013; García Nieto, 2011; McFarlane, 2016; Yale, 2019), from which multiple dimensions of analysis derive. In this respect, works such as those by López-Gómez (2015, 2017), León (2018), and Martínez Clares, Martínez Juárez, and Pérez Cusó (2016) provide a broad review and clear vision of the future of the term in order to consider how it is understood and how it evolves. This allows us to find the definition of "tutoring" as an educational process university teachers, based on the premises of orientation that reacts integrally to students' needs (academic, social-personal, and/or professional) with the objective of fostering the optimal teaching and learning process.

On the basis of this conception, we can identify three clearly defining dimensions to which this work contributes, which relate to the tutor's work (profile and functions) and to students' tutorial needs.

\section{Functions and profiles of the university teacher-tutor}

At a regulatory level, section 8 of Spain's University Students' Charter (Royal Decree 1791/2010, of 30 December) establishes a right to «receive personalised orientation and tutoring to facilitate workplace integration, professional development, and, where applicable, the continuity of university education.» Section 20 expressly states that the teachertutor is responsible for this service and must provide it on three levels (when starting university, during university studies, and in the transition to the world of work). Based on this, multiple works consider and evaluate the tutor's professional activity with the aim of laying the foundations for appropriate tutorial action (Álvarez-Pérez, López, \& Pérez, 2016; Arza, Salvador, \& Mascarenhas, 2014; Bisquerra, 2013; Gaitán, 2013; García-Valcárcel, 2008; López-Gómez, 2017; Martínez Clares et al., 2016; Torrecilla, Rodríguez, Herrera, \& Martín, 2013; Troyano \& García, 2009; Urbina, de la Calleja, \& Medina, 2017).

Having considered the focuses on tutorial action that derive from these works, we use the structure proposed by Lobato and Guerra (2016), which identifies seven categories of university tutoring, five of which are explicitly carried out by university teachers. In turn, following on from the work of Rodríguez Espinar (2004), complemented by Álvarez González (2013, 2014) and Álvarez González and Álvarez (2015), we believe that the set of duties of a university tutor are based on three models of tutorial action (Table 1). 
TABLE 1. Types of tutoring in universities

\begin{tabular}{|c|c|c|c|}
\hline Type & Definition of functions & Figure & System \\
\hline $\begin{array}{l}\text { Academic } \\
\text { tutoring }\end{array}$ & $\begin{array}{l}\text { Monitoring and supporting the lear- } \\
\text { ning process in a module or subject } \\
\text { (dealing with problems that arise; } \\
\text { making working methodologies more } \\
\text { flexible; developing evaluation pro- } \\
\text { ducts; promoting the development of } \\
\text { research work; establishing effective } \\
\text { communication; making use of criti- } \\
\text { cal behaviour and engagement, etc.). }\end{array}$ & \multirow[t]{3}{*}{ Teacher } & $\begin{array}{l}\text { Tutorial Action } \\
\text { Plan (TAP) of } \\
\text { the university or } \\
\text { faculty. }\end{array}$ \\
\hline $\begin{array}{l}\text { Work } \\
\text { placement } \\
\text { tutoring }\end{array}$ & $\begin{array}{l}\text { Advising students during their work } \\
\text { placement period. }\end{array}$ & & $\begin{array}{l}\text { Work placement } \\
\text { programme }\end{array}$ \\
\hline $\begin{array}{l}\text { Research } \\
\text { tutoring }\end{array}$ & $\begin{array}{l}\text { Individualised guidance for prepa- } \\
\text { ring a research project during un- } \\
\text { dergraduate studies and all levels of } \\
\text { postgraduate study. }\end{array}$ & & $\begin{array}{l}\text { Planned between } \\
\text { teacher and stu- } \\
\text { dent }\end{array}$ \\
\hline $\begin{array}{l}\text { Personal } \\
\text { tutoring }\end{array}$ & $\begin{array}{l}\text { Individualised and specialised atten- } \\
\text { tion to resolve personal difficulties } \\
\text { that affect academic performance } \\
\text { (helping students with the process of } \\
\text { developing self-knowledge; guidance } \\
\text { with the process of taking personal } \\
\text { decisions; help with conflict resolu- } \\
\text { tion; encouraging the development of } \\
\text { personal skills; etc.). }\end{array}$ & $\begin{array}{l}\text { Specialist } \\
\text { professional / } \\
\text { teacher }\end{array}$ & $\begin{array}{l}\text { At student's } \\
\text { request }\end{array}$ \\
\hline $\begin{array}{l}\text { Degree or } \\
\text { course tuto- } \\
\text { ring }\end{array}$ & $\begin{array}{l}\text { Intervention to support personal, aca- } \\
\text { demic, and professional development } \\
\text { throughout the student's time at uni- } \\
\text { versity (knowledge of the student's } \\
\text { profile; supporting the student's } \\
\text { process of integration; contributing } \\
\text { to the student's academic-social inte- } \\
\text { gration; providing curriculum infor- } \\
\text { mation and orientation; etc.). }\end{array}$ & Teacher & TAP \\
\hline Peer tutoring & $\begin{array}{l}\text { Experienced student who supports } \\
\text { students on same course. }\end{array}$ & Student & TAP \\
\hline $\begin{array}{l}\text { Service tuto- } \\
\text { ring }\end{array}$ & $\begin{array}{l}\text { Support comprising academic and ca- } \\
\text { reers information and guidance for all } \\
\text { of a university's student body. }\end{array}$ & Specialists & $\begin{array}{l}\text { Institutional } \\
\text { service }\end{array}$ \\
\hline
\end{tabular}

Source: Adapted from Lobato and Guerra (2016, p. 385).

To implement these functions, García Cabrero et al. (2016) suggest that the tutor must acquire skills while simultaneously being familiar with institutio- nal information, as providing a service in line with the needs of students will largely require this level of professionalisation. Therefore, it is necessary to 
consider the role adopted by university teachers (attitudes) with regards to the importance attributed to tutorial activities, being clear, as Más Torelló (2012, p. 303) notes, that «tutoring the student's learning process by facilitating actions that allow him or her greater autonomy» must be one of the basic competences in the tutor's professional profile.

In this regard, educational research (Fernández-Salinero, 2014; GonzálezBenito \& Vélaz de Medrano, 2014; González-Lorente \& González-Morga, 2015; Herrera Rodríguez, 2017; López, González, \& Velasco, 2013; Martínez Clares, Martínez Juárez, \& Pérez Cusó, 2014; Martínez-Mínguez \& Moya, 2017) has identified common features relating to this role and contributed to shaping recognisable professional profiles, which have specific attributes relating to competences that include «certain skills, appropriate knowledge, and a particular attitude, guided by ethical principles» (Giner, 2012, p. 28). This shapes a broad spectrum of approaches to tutorial activity in universities, synthesised in the paternalistic model (pastoral care) and a more distant model (laissez-faire) as the extremes from which different degrees of involvement, action, and commitment are assumed (Cid \& Pérez, 2006).

In general terms, in accordance with works by Echeverría (2002), García Nieto, Asensio Muñoz, Carballo Santaolalla, García García and Guardia González (2004), and Lázaro (2003), we can establish a classification that considers the tutor's areas of activity and which, depending on how a given tutor is positioned on the continuum between the models identified, enables us to speak of a human profile and professional profile. The former includes the tutor's being and knowing how to be, encompassing desirable personal qualities such as:

a) A balanced healthy mature personality through which positive attitudes are developed and target actions are proposed to ensure equanimity and fairness in decisions (without apparent preferences or aversions in treatment).

b) A sensitivity that makes it possible to perceive and understand youth problems, fostering empathetic and affective behaviour patterns, creating an atmosphere of positive care, and making respect for the student apparent, accompanied by a calm attitude in the decisions taken.

c) The ability to establish friendly and cordial relationships with students from which the tutor can glean knowledge of the group and the individuals, making it possible to have a positive influence on their opinions and attitudes.

The professional profile relates to the tutor's knowledge and know-how in his or her command of this comprehension and exercise of the tutorial action. Regarding comprehension of the tutorial activity, it is desirable to have: 
a) A subject knowledge that allows the tutor to explain competently the subjects he or she delivers.

b) Theoretical knowledge of education and related sciences. In particular, an in-depth knowledge of the general features of the university system (pathways, specialisms, career prospects, etc.) and also the characteristics of those academic fields that comprise it (psychology, teaching, philosophy, sociology, etc.) is desirable.

c) A theoretical-practical comprehension of interpersonal relationships and of leading and encouraging groups so that the tutor can identify structures, roles, factors, or forces that affect the development of the group of students.

d) A knowledge of techniques for educational diagnosis and intervention to provide more personal attention, foster motivation, and promote initiativesprogrammes relating to attention to diversity.

With regards to the practical exercise of tutoring activities, it would also be desirable to implement techniques relating to evaluation, carrying out interviews, invigorating groups, promoting social skills, motivating, etc.

We believe that the best university tutor profile will combine a balance of the different competences, creating what Hagenauer and Volet (2014) call the support dimension or atmosphere of su- pport, in which the student perceives a personalised process of accompaniment throughout his or her time at university and, at the same time, feels that his or her needs are being met.

\section{Tutorial needs of university students}

The orientation needs that university tutoring must meet could be analysed on the basis of the three phases associated with students' time at university (entry, performing their studies, and the transition to the world of work).

If we start by considering the moments when the student first comes into contact with universitylevel education, we find many studies (Da Re \& Clerici, 2017; Esteban, Bernardo, \& Rodríguez-Muñiz, 2016; García-Ros, Pérez-González, Pérez-Blasco, \& Natividad, 2012; García, Carpintero, Biencinto, \& Núñez, 2014; López Gómez, 2015; Lorenzo Moledo, Argos, Hernández García y Vera Vila, 2013; Manzano-Soto \& Roldán-Morales, 2015; Pujol \& Durán, 2013; Rebollo \& Espiñeira, 2017; Soares, Almeida, \& Guisande, 2011; Torrecilla et al., 2013). These studies can form the basis for a focus on the demands associated with three dimensions:

Academic (unfamiliarity with the institution; skills imbalances when faced with high demands; problems managing study time; stress in especially busy periods; etc.).

Personal (lack of self-regulation skills; shortcomings in the development of de- 
cision making; lack of autonomy; strengthening metacognitive strategies; boosting motivation to study; etc.).

Social (difficulties creating new social networks; coping with separation from family ties; difficulties combining studying and work; improving social skills, and managing conflict in the classroom; etc.).

Some of the needs identified here might continue in later phases (carrying out studies and transition to the world of work) and other new needs can emerge (Figuera \& González, 2014; Martínez Clares et al., 2019; Pérez, González, González, \& Martínez, 2017; SolagurenBeascoa \& Moreno, 2016):

- At an academic level, demands focus on two main related areas for supporting the teaching-learning process (knowledge of study techniques; orientation in final degree projects; knowledge of the working dynamics-methodologies of teaching staff; better understanding of syllabuses, such as optional courses, characteristics of the modules, system of credits; problems with the range of evaluation systems, etc.) and for supporting professional development and social and job market inclusion (lack of knowledge of career prospects; guidance with decision making when facing the transition to the job market; the demand for opportunities for developing professional skills; better knowledge of postgraduate training, etc.).
- At a personal level, the need to build a consolidated identity intensifies (managing emotions; acquiring transversal competences; support with the process of personal maturation and in setting future goals; etc.).

- At a social level, it affects aspects pertaining to the development of interpersonal relationships (helpmediation with conflict management; improving communication skills; guidance in developing social skills; facilitating strategies for team working; etc.).

In summary, we believe that to offer an optimal response to these needs, university tutoring must be considered from a holistic and integral position which, at the same time, is adapted to the contexts in which the students find themselves (López \& González, 2018).

\section{Research approach, aims, ob- jectives, and hypothesis}

As a general aim, we intend to analyse the effect of the profile and functions of the tutor on higher-education students' tutorial needs. To achieve this, we propose the following objectives:

- Validation of a scale for evaluating tutoring in higher education.

- Validation of the construct of the interrelation of factors associated with tutoring in higher education (tutor profile, tutor functions, and tutorial needs). 
- Confirmation of the positive influence of the factors associated with a tutor's profile on his or her functions and on the tutorial needs of higher-education students, and the influence of these tutor functions on the tutorial needs of the student.
In addition, based on the theoretical foundations, and as a consequence of the proposed objectives, we design a model which we subject to empirical evaluation (Graph 1):

GRAPH 1. Structural model to be confirmed.

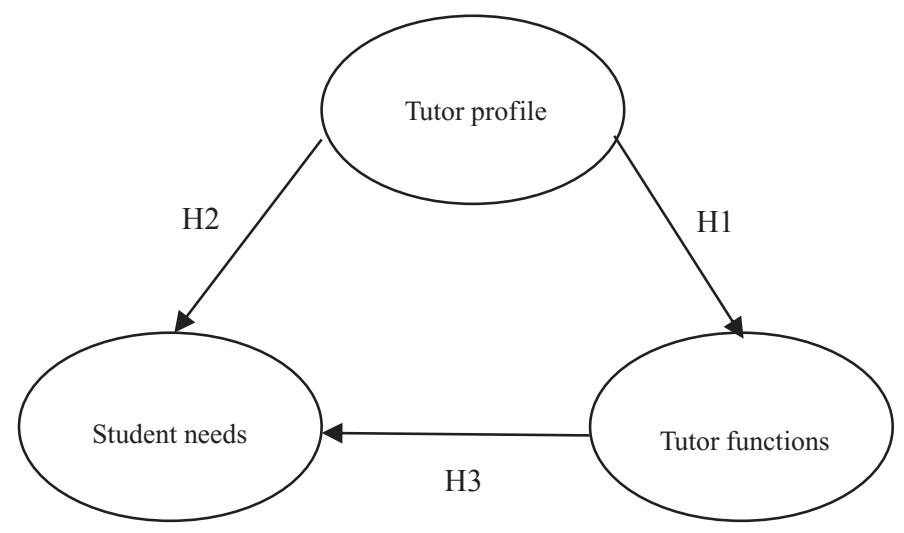

Source: Own elaboration.

Following the reference model, the proposed hypotheses are:

- Hypothesis 1 (H1): the tutor profile predicts the type of functions he or she performs (Álvarez González, 2013, 2014; Cid \& Pérez, 2006; Rodríguez Espinar, 2004).

- Hypothesis 2 (H2): the tutor profile predicts the effect he or she has on students' tutorial needs (García Nieto et al., 2004; López \& González, 2018; Martínez Clares, et al., 2019).

- Hypothesis 3 (H3): the tutor functions have a direct influence on the effect on the tutorial needs of the students
(Amor Almedina, 2016; López \& González, 2018; Urbina et al., 2017).

\section{Methodology}

This research uses a survey-type methodological design intended to verify a theoretical model of the interrelation of different factors associated with tutoring in higher education. We used the structural equation technique for this purpose, using the Amos 18.0 program.

\subsection{Participants}

We carried out convenience nonprobability sampling on the study population (students enrolled on undergraduate 
degree courses at the Universidad de Huelva) in which we identified the representativeness of students enrolled on courses in different areas of knowledge and gender diversity as quotas. We performed this procedure on a sample of 581 students $(49.1 \%$ male; $50.9 \%$ female, mean age 22.94 years) enrolled in various academic years $(35.5 \%$ in the first year; $27.5 \%$ in the second year; $28.1 \%$ in the third year; $9 \%$ in the final year) in 30 different degree courses (arts and humanities [4.13\%], sciences [3.09\%], health sciences [8.77\%], social and legal sciences [61.27\%], and engineering and architecture [22.71\%]). This size is sufficient (95\% confidence level; $5 \%$ sampling error), although it is not optimal for initial validation.

\subsection{Instrument}

For data collection we used an ad hoc scale (see Table 2) with three dimen- sions (Tutor Functions [TF], Tutor Profile [TP], and Students' Tutorial Needs [TN]) with 26,13 , and 21 items respectively, set out on a Likert-type scale with values from 1 to 7 , with 1 being not at all important and 7 very important. We prepared the instrument based on the reference theoretical foundations from the literature we consulted (Álvarez González, 2013, 2014; Amor Almedina, 2016; Cid \& Pérez, 2006; Echeverría, 2002; García Nieto et al., 2004; Lázaro, 2003; López \& González, 2018; Martínez Clares et al., 2019; Rodríguez Espinar, 2004, among others) and which sketch out the profile of the tutor and the functions he or she performs as factors associated with satisfactory tutoring. From this, we can establish the tutorial needs of university students and react to them.

TABLE 2. Scale for evaluating tutoring in higher education.

\section{Tutor (and tutoring) functions for students (TF)}

F1. Informing students about institutional academic questions (administrative organization, syllabus, modules, regulations governing progression, pathways, etc.).

F2. Providing institutional support to newly enrolled students.

F3. Guaranteeing personalised academic attention.

F4. Orientation and guidance on teaching-learning strategies.

F5. Orientation and guidance on learning styles, intellectual working techniques, and study habits.

F6. Orientation about developing individual study plans.

F7. Orientation about developing generic competences.

F8. Monitoring and evaluation of students, giving them information and guidance on their results and performance.

F9. Guidance on preparing course work.

F10. Guidance on and recommending bibliographies for the subject in his or her specialist area.

F11. Guidance on taking part in university life.

F12. Mediation in cases of conflict (teacher/student; student/student; student/institution).

F13. Orientation about social and communication skills. 
F14. Orientation about interpersonal competences.

F15. Orientation about solving personal, family, health, social, etc. conflicts.

F16. Guidance on affective relationships.

F17. Orientation about decision making.

F18. Orientation about career prospects, workplace integration, and the job market.

F19. Guidance on developing professional skills.

F20. Providing professional references.

F21. Orientation and guidance about social questions (housing, grants, cultural context, etc.).

F22. Diagnosing and evaluating individual and group educational needs.

F23. Coordinating or liaising with the teaching team.

F24. Coordinating with faculty/university authorities and with support staff (administration and service staff, scholarships assistants, etc.).

F25. Supporting students with Erasmus/Socrates grants.

F26. Coordinating and supervising students' work placements in businesses/institutions.

\section{Human and professional profile of the tutor (TP)}

P1. Affectivity.

P2. Empathy.

P3. Personalisation (personally knowing each student).

P4. Fairness, equanimity (no preferences or aversions).

P5. Calm authority (ability to control a group, command respect, not authoritarianism).

P6. Respect for students.

P7. A personality that can positively influence and convince.

P8. Capacity to relate to others.

P9. Ability to listen.

P10. Receptive attitude and willingness to dialogue.

P11. Subject knowledge (academic knowledge).

P12. Knowledge of educational sciences (knowledge of teaching).

P13. Knowledge of social relationships and group dynamics.

\section{Tutorial needs of students (TN)}

N1. Orientation for the transition from secondary education to university.

N2. Orientation on arrival at university (first year).

N3. Helping students combine work and studying.

N4. Orientation for exams.

N5. Reviewing exams.

N6. Orientation after evaluations.

N7. Orientation during the progress of modules.

N8. Assistance with doubts about the subject.

N9. Orientation about improving academic performance. 
N10. Orientation about factors linked to age (young people, adults, older people, etc.).

N11. Orientation about questions of cultural diversity (ethnicity, country, social group, etc.).

N12. Orientation about questions of linguistic diversity.

N13. Orientation for gifted and talented students.

N14. Orientation about special educational needs.

N15. Orientation about visual, auditory, motor, etc. disabilities.

N16. Orientation and evaluation of aptitudes (capacities).

N17. Orientation and evaluation of attitudes and personality.

N18. Orientation and evaluation of learning styles.

N19. Orientation and evaluation of cognitive strategies.

N20. Orientation and evaluation of professional interests.

N21. Orientation and evaluation of motivation.

Source: Own elaboration.

To collect data, we distributed the instrument to students individually for a duration of approximately 15 minutes. We informed the students that participation was voluntary and explained the instructions and objectives of the survey to them. We also told them it was confidential. We used the cross-sectional bands of tutorials so that we could access the population without having to interfere in academic rhythms. Finally, we used the SPSS 23 computer program for statistical analysis of the data obtained.

\section{Results}

\subsection{Validation of the instrument}

As mentioned above, we designed the instrument specially for this piece of research based on the works cited. We then submitted it for expert evaluation (six academics specialising in education at the Universidad de Huelva). This enabled us to evaluate the pertinence and clarity of the items. Modifications to the initial ver- sion were minor. Both processes contribute to the theoretical validity.

\subsubsection{Reliability}

Applying Cronbach's Alpha to estimate the internal consistency of the scale, which has 60 variables and a sample of 581 subjects, showed a high level of reliability $(\alpha=.953)$ for the complete scale. We also found high partial correlations for each dimension (TF, $\alpha=.919$; TP, $\alpha=.889$; TN, $\alpha=.921)$. Next we identified the less consistent items to revise the scale (items F6 and item N1) and improve it for future applications. When we excluded these, the total alpha value was .958 and two of the three dimensions also improved (TP, $\alpha=.928 ; \mathrm{TN}, \alpha=.929$ ).

\subsubsection{Construct validity}

We performed an exploratory factor analysis (EFA) with the aim of concentrating the relevance of the items on one factor and so better discriminating between factors. We performed a factorial reduc- 
tion applying an orthogonal rotation using the varimax method. Furthermore, in view of the high KMO values for the scales - between .908 and .937 - and the level of significance of $<.001$ obtained in the Bartlett sphericity test, we can confirm that factor analysis is relevant, suitable, and applicable.

The results for each of the dimensions considered are set out below:

D1. Tutor's functions regarding students: we identified three factors that explain $53.33 \%$ of the variance in the set of items (Table 3):
- Factor 1: general guidance and orientation functions. The items saturated by this factor include: those relating to orientation about skills, competences, career prospects, conflict resolution, decision making, social questions, and learning styles; and those that refer to guidance in professional competences, affective relationships, teaching - learning strategies, and university participation. They also include functions regarding evaluating and diagnosing educational needs and functions related to coordinating the teaching team and coordinating with authorities (faculty/university).

TABLE 3. Factorial analysis of tutor functions.

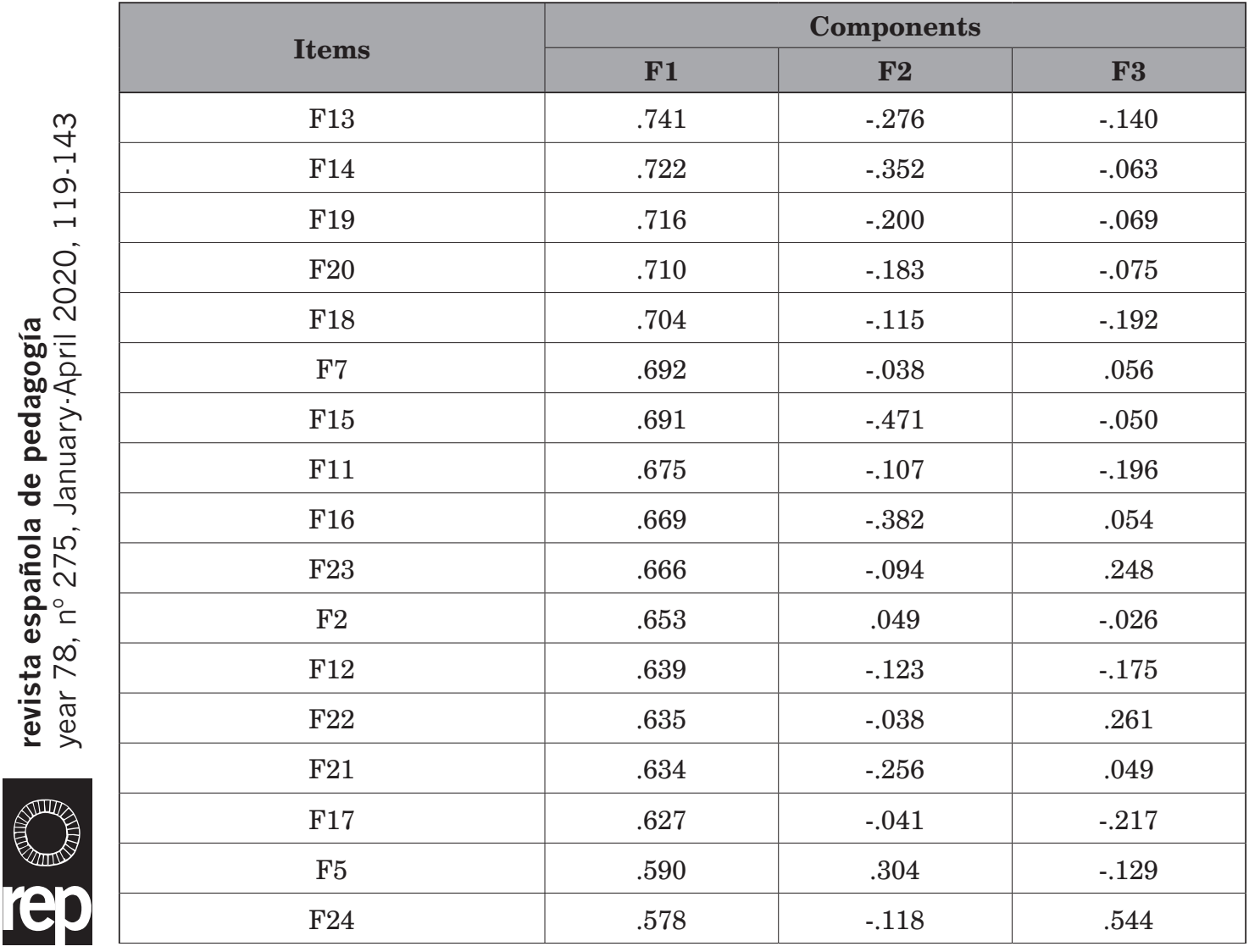


Profiles and functions of university tutors and their effects on students' tutorial needs

\begin{tabular}{|c|c|c|c|}
\hline F4 & .541 & .472 & -.208 \\
\hline F1 & .514 & .436 & -.041 \\
\hline F10 & .466 & .403 & -.118 \\
\hline F9 & .384 & .667 & -.114 \\
\hline F8 & .422 & .590 & -.163 \\
\hline F3 & .480 & .523 & -.033 \\
\hline F26 & .452 & .287 & .561 \\
\hline F25 & .417 & .360 & .498 \\
\hline Variance explained by factor & 37.236 & 10.892 & 5.210 \\
\hline
\end{tabular}

Rotated component matrix $(\mathrm{KMO}=.917$; Bartlett. $\mathrm{Sig}=.000)$.

Source: Own elaboration.

- Factor 2: personalised student monitoring functions. This factor saturates variables that illustrate the functions of the tutor such as guidance on coursework, monitoring and evaluation of students, and personalised academic attention.

- Factor 3: functions relating to work placements and Erasmus grants. This factor saturates variables that illustrate the coordination and supervision of work placements and support for students with Erasmus/Socrates grants.
D2. Tutor profile: we identified two factors that explain $56,254 \%$ of the variance in the set of items (Table 4). Namely:

- Factor 1: professional profile. This factor illustrates variables relating to the capacity to listen and relate to others, characteristics such as empathy, leadership, equanimity, and authority, while also showing knowledge of education and subject knowledge.

TABLE 4. Factorial analysis of the tutor profile.

\begin{tabular}{|c|c|c|}
\hline \multirow{2}{*}{ Items } & \multicolumn{2}{|c|}{ Components } \\
\cline { 2 - 3 } & F1 & F2 \\
\hline P6 & .783 & .177 \\
\hline P9 & .763 & .182 \\
\hline P11 & .759 & .038 \\
\hline P12 & .736 & .030 \\
\hline P4 & .710 & .314 \\
\hline P5 & .692 & .159 \\
\hline P7 & .641 & .242 \\
\hline P10 & .570 & .374 \\
\hline
\end{tabular}


Manuel DELGADO-GARCÍA, Sara CONDE VÉLEZ and Ángel BOZA CARREÑO

\begin{tabular}{|c|c|c|}
\hline P2 & .540 & .439 \\
\hline P8 & .497 & .457 \\
\hline P1 & .051 & .882 \\
\hline P3 & .100 & .873 \\
\hline P13 & .435 & .459 \\
\hline Variance explained by factor & 44.591 & 11.663 \\
\hline
\end{tabular}

Rotated component matrix $(\mathrm{KMO}=.908$; Bartlett. Sig $=.000)$.

Source: Own elaboration.

- Factor 2: human and social profile. - Factor 1: needs that focus on personal This factor characterises a sensitive tutor profile, concerned with getting to know each student individually and knowledge of social relationships and group dynamics.

D3. Students' tutorial needs: three factors are identified that explain $61.029 \%$ of the variance in the set of items (Table 5). and/or professional aspects. This factor brings together a group of items that emphasise the importance of needs for orientation and guidance on learning styles, attitudes, and personality, cognitive strategies, motivation, and professional interests and aptitudes.

Namely:

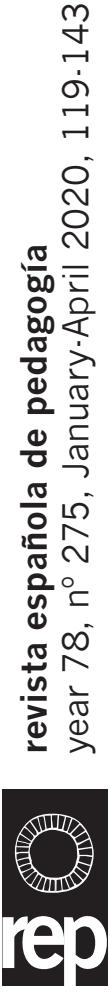

TABLE 5. Factorial analysis of students' tutorial needs.

\begin{tabular}{|c|c|c|c|}
\hline \multirow{2}{*}{ Items } & \multicolumn{3}{|c|}{ Components } \\
\cline { 2 - 4 } $\mathrm{N} 18$ & $\mathbf{1}$ & $\mathbf{2}$ & $\mathbf{3}$ \\
\hline $\mathrm{N} 17$ & .763 & .180 & .176 \\
\hline $\mathrm{N} 19$ & .733 & .371 & .113 \\
\hline $\mathrm{N} 21$ & .717 & .254 & .220 \\
\hline $\mathrm{N} 20$ & .707 & .394 & .093 \\
\hline $\mathrm{N} 16$ & .698 & .330 & .146 \\
\hline $\mathrm{N} 12$ & .615 & .465 & .133 \\
\hline $\mathrm{N} 11$ & .259 & .795 & .169 \\
\hline $\mathrm{N} 13$ & .197 & .761 & .053 \\
\hline $\mathrm{N} 10$ & .328 & .716 & .100 \\
\hline
\end{tabular}


Profiles and functions of university tutors and their effects on students' tutorial needs

\begin{tabular}{|c|c|c|c|}
\hline $\mathrm{N} 3$ & .312 & .585 & .279 \\
\hline $\mathrm{N} 15$ & .462 & .575 & .246 \\
\hline $\mathrm{N} 14$ & .506 & .554 & .154 \\
\hline $\mathrm{N} 2$ & .359 & .483 & .402 \\
\hline $\mathrm{N} 5$ & .037 & .129 & .802 \\
\hline $\mathrm{N} 8$ & .067 & .158 & .770 \\
\hline $\mathrm{N} 6$ & .157 & -.020 & .743 \\
\hline $\mathrm{N} 7$ & .264 & -.006 & .743 \\
\hline $\mathrm{N} 4$ & .047 & .225 & .736 \\
\hline $\mathrm{N} 9$ & .309 & .353 & .536 \\
\hline Variance explained by factor & 43.446 & 12.588 & 5.001 \\
\hline
\end{tabular}

Rotated component matrix $(\mathrm{KMO}=.937$; Bartlett. $\mathrm{Sig}=.000)$

Source: Own elaboration.

- Factor 2: needs that focus on attention to diversity. This factor illustrates needs that relate to attention to diversity: linguistic, cultural, age, disabilities, and special educational needs.

- Factor 3: needs that focus on evaluation and performance in modules. This factor saturates a series of needs whose importance centres on reviewing exams, doubts about the subject, and needs for orientation focussing on the evaluation processes (before, during, and after) with orientation for improving academic performance.

\subsection{Confirmation of the model}

After the preliminary reduction of the number of factors, we attempted to

empirically test the factors extracted. To model the structural equation, we took as reference points the hypotheses stated in the theoretical model that guides the research (Graph 1).

The high rates of variance explained in the factors relating to the general orientation and guidance functions (62\%), personalised student-monitoring functions $(42 \%)$, functions relating to work placements and Erasmus grants $(87 \%)$, needs centred on personal and/or professional aspects (92\%), needs centred on attention to diversity (87\%), and needs centred on evaluation and performance in modules (98\%), and the regression and correlation indexes between the factors included in the equation allow us to accept the model (Graph 2). 
Graph 2. Structural Equation Model.

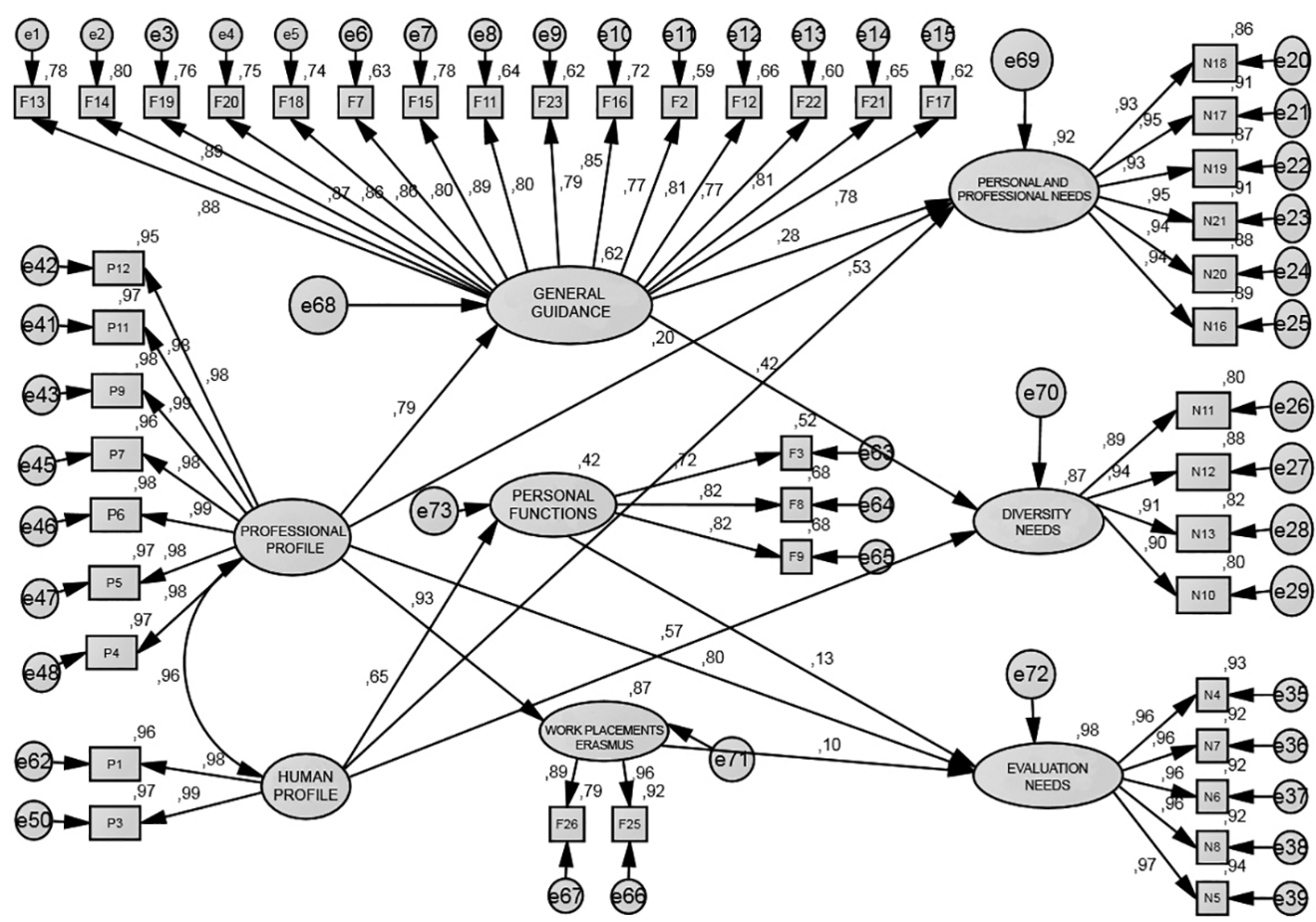

Source: Own elaboration.

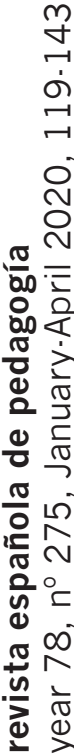

cate that the model fits the data reasonably $\quad \mathrm{IFI}=.93 ; \mathrm{NFI}=.98$; $\mathrm{RMSEA}=.04$ ).

TABLE 6. Fit indices of the model.

\begin{tabular}{|l|l|c|c|}
\hline \multicolumn{2}{|c|}{ Fit indices } & $\begin{array}{c}\text { Recommended } \\
\text { values }\end{array}$ & $\begin{array}{c}\text { Observed } \\
\text { values }\end{array}$ \\
\hline Absolute fit index & $\chi^{2} /$ df (Chi-squared/df) & $\leq 5.00$ & $4.76 ; p<.001$ \\
\hline \multirow{2}{*}{ Comparative fit index } & IFI (incremental fit index) & $\geq .90$ & .93 \\
\cline { 2 - 4 } & NFI (normalised fit index) & $\geq .90$ & .98 \\
\cline { 2 - 4 } & CFI (comparative fit index) & $\geq .90$ & .93 \\
\hline \multirow{2}{*}{ Error of approximation } & $\begin{array}{l}\text { RMSEA (Root Mean Square } \\
\text { Error of Approximation) }\end{array}$ & $\leq .06$ & .04 \\
\hline Sample fit & HOELTER .05 & $>200$ & 480 \\
\hline
\end{tabular}

Source: Own elaboration. 
In the model we can see three groups of elements: firstly, the independent factors that relate to the tutor profile (social and professional); secondly, as dependent variables, the functions tutors perform measured in three types (general orientation and guidance, personalised orientation and guidance, and orientation and guidance relating to work placements and Erasmus/Socrates grants); and thirdly, the tutorial needs of the students (regarding personal and/or professional aspects; focussed on attention to diversity; relating to evaluation and performance in modules).

The model proposes correlations between the tutor profile, and regressions of the influences of these profiles on the functions and needs. Therefore, starting with the correlations between the independent factors, we identified a strong correlation between the human and social profile and the professional profile $(\mathrm{r}=.96 ; p \leq .01)$ and, analysing in more depth the regression indices between factors, we found the following results:

Depending on the type of profile associated with the tutor, we can predict the type of functions he or she performs, as well as the tutorial needs of the students he or she looks after; accordingly, in the model we can see how a more professional tutor profile has a significant positive influence on the general guidance and orientation functions $(\beta=.79 ; p \leq .01)$ and
Erasmus placement function $(\beta=.93$; $p \leq .01$ ), and reacts to a type of needs focussed both on personal/professional aspects $(\beta=.20 ; p \leq .01)$ and on the students' needs relating to evaluation and performance $(\beta=.80 ; p \leq .01)$. We also see how a more human/social profile predicts more personalised functions relating to the monitoring of students $(\beta=.65 ; p \leq .01)$ and taking care of personal, professional $(\beta=.53 ; p \leq .01)$ and diversity needs $(\beta=.57 ; p \leq .01)$.

With regards to functions, we can see how, depending on the typology developed, their influence on tutorial needs is positive, and on a different level, these needs are also shaped by the profile that defines the tutor. In this way, we can see how the general orientation and advising functions have a positive and significant influence on needs that centre on personal and/or professional aspects $(\beta=.28 ; p \leq .01)$, and on those connected to attention to diversity $(\beta=.42 ; p \leq .01)$.

Furthermore, the needs that focus on evaluation and monitoring of modules are predicted by personalised functions relating to monitoring students $(\beta=.13$; $p \leq .01$ ) and, at a lower level, by functions relating to work placements and Erasmus grants $(\beta=.10 ; p \leq .01)$.

Finally, regarding the measurement model, for those factors that saturate a large number of indicators, we decided to include the items with factor loadings above .6 as a better fit is achieved (Table 7): 
TABLE 7. Factor loadings of the measurement model.

\begin{tabular}{|c|c|}
\hline Indicator & Estimate \\
\hline \multicolumn{2}{|l|}{ GENERAL ADVICE } \\
\hline F13 & .885 \\
\hline F14 & .893 \\
\hline F19 & .870 \\
\hline F20 & .863 \\
\hline F18 & .858 \\
\hline F7 & .796 \\
\hline F15 & .886 \\
\hline F11 & .801 \\
\hline F23 & .785 \\
\hline F16 & .851 \\
\hline $\mathrm{F} 2$ & .768 \\
\hline F12 & .813 \\
\hline F22 & .772 \\
\hline F21 & .806 \\
\hline F17 & .785 \\
\hline \multicolumn{2}{|c|}{ PERSONAL AND PROFESSIONAL NEEDS } \\
\hline N18 & .926 \\
\hline N17 & .955 \\
\hline N19 & .935 \\
\hline $\mathrm{N} 21$ & .952 \\
\hline N20 & .937 \\
\hline N16 & .942 \\
\hline \multicolumn{2}{|l|}{ DIVERSITY NEEDS } \\
\hline N11 & .893 \\
\hline $\mathrm{N} 12$ & .938 \\
\hline N13 & .906 \\
\hline N10 & .896 \\
\hline
\end{tabular}


Profiles and functions of university tutors and their effects on students' tutorial needs

\begin{tabular}{|c|c|}
\hline \multicolumn{2}{|l|}{ EVALUATION NEEDS } \\
\hline $\mathrm{N} 4$ & .965 \\
\hline N7 & .959 \\
\hline N6 & .957 \\
\hline N8 & .961 \\
\hline N5 & .972 \\
\hline \multicolumn{2}{|l|}{ PROFESSIONAL PROFILE } \\
\hline P11 & .983 \\
\hline $\mathrm{P} 12$ & .977 \\
\hline P9 & .987 \\
\hline $\mathrm{P} 7$ & .979 \\
\hline $\mathrm{P} 6$ & .990 \\
\hline P5 & .983 \\
\hline $\mathrm{P} 4$ & .985 \\
\hline \multicolumn{2}{|l|}{ HUMAN PROFILE } \\
\hline P3 & .987 \\
\hline $\mathrm{P} 1$ & .980 \\
\hline \multicolumn{2}{|l|}{ PERSONAL FUNCTIONS } \\
\hline F3 & .720 \\
\hline F8 & .824 \\
\hline F9 & .824 \\
\hline \multicolumn{2}{|l|}{ ERASMUS PLACEMENTS } \\
\hline F26 & .888 \\
\hline F25 & .960 \\
\hline
\end{tabular}

Source: Own elaboration.

7. Discussion and Conclusions lidation of an instrument and of the consThe aim of this work was to analyse truct being studied, in order to achieve a how tutor profiles and functions impact valid and reliable analysis of the results the tutorial needs of higher education obtained, which the EFA confirmed. The students. To this end, we identified two model was then confirmed using the strucspecific objectives that constrained the va- tural equations procedure, which leads us 
to identify the existence of correlations between the tutor profile, and regressions or influences of these profiles on their own functions and on the needs of the students.

By specifying the objectives and examining the stated hypotheses, the results allow us to confirm these hypotheses through various demonstrated correlations. The following conclusions stand out:

a) There is a university teacher professional profile that prioritises the academic area of the tutorial activity with the aim of meeting students' needs that relate to academic evaluation and performance; this requires the tutor to demonstrate certain professional competences (the ability to listen and relate to people, and to display knowledge of education and the subject he or she delivers) and personality traits (empathy, leadership, equanimity, and authority) which are also illustrated in other pieces of research (Fernández-Salinero, 2014; García Cabrero, et al., 2016; Martínez Clares etal., 2014; Más Torelló, 2012; Torrecilla et al., 2013). This tutor profile is also linked to performing functions that are personalised and relate to monitoring of students (guidance with coursework, monitoring and evaluating the student body, and personalised academic attention), and to those functions linked to guidance in work-placement periods and/or on grant programmes (Erasmus/Socrates).

b) There is also a humanitarian profile of a university teacher who carries out the personal and social area of the tu- torial activity more intensely, in order to react to student needs that are closely linked to highly personal aspects of their personality, taking professional decisions, and ensuring attention to diversity; this requires the tutor to display sensitive behaviour, an interest in the personalisation and individualisation of teaching, and also to be involved in the social and dynamic relationships of groups. This tutor profile carries out functions relating to orientation and general guidance (orientation about skills, competences, career prospects, conflict resolution, decision making, social questions, learning styles, guidance on professional competences, affective relationships, teaching-learning and university participation strategies). Although this profile does not have the same long history in Spain as it does in some other places like the English-speaking world (Cashmore, Scott, \& Cane, 2012, analysed the importance of the role of the tutor in creating a feeling of belonging to the institution and avoiding early university leaving), it is currently emerging as one of the central functions in university tutoring, and so it is necessary to consider in greater depth the principles that should guide its implementation (Haya, Calvo, \& Rodríguez, 2013; Herrera Rodríguez, 2017; López \& González, 2018; López et al., 2013; Manzano-Soto \& Roldán-Morales, 2015; Martínez Clares et al., 2019; Yale, 2019).

Two models of tutorial action are apparent:academic-professional (teaching) and 
personal (counselling). These are defined by the needs students display, which polarise the work and profile of university tutors, and so this piece of work supports the existing theoretical theses that shape the different classifications of university tutoring models (Álvarez González, 2013, 2014; Álvarez González \& Álvarez, 2015; Lobato \& Guerra, 2016) and it also underlines the need to take students' needs into consideration as a starting point from which to design and implement the tutorial practices included in the different services and/or programmes offered by the university.

To meet this challenge, the study presented here agrees with other recent works (Martínez Clares et al., 2019; Waltz, 2019) on the importance of providing teachers with information and practical training to create a «professional awareness" that can form the basis for acquiring a commitment to a function that is inherent to teaching practice and «must not be based solely on technical and methodological aspects, but also on the personal and participatory competences that tutoring demands» (Martínez Clares et al., 2016, p. 95). Faced with crowded settings that can tend to depersonalise the process of teaching and learning, students clearly express concerns about this, and works such as those by Amor Almedina (2016), Cashmore et al. (2012), López Gómez (2017), and Pérez et al. (2017) show that tutors being closer to the students results in a process whereby students better adapt to the university, develop a sense of belonging at the institution, and increa- se their expectations of academic success and achievement.

In short, the proposed integral tutorial activity (Álvarez González, 2017) must be incorporated into the work of the university tutor, something demonstrated in much of the theory which acts as a foundation for the present study and in the results obtained in this study.

\section{Limitations and future lines of research}

Among this study's limitations, we should note the need to compare the opinion of students with that of the university tutor to achieve a better fit between, and significance of, the proposed objectives. Also, increasing the size of the final sample in some of the subject areas would make for more generalisable information that starts from a concrete reality but is similar to other contexts analysed in the rationale for the study.

Looking to the future, this work supports the need to continue to investigate the needs of university students and teachers' work as tutors, to create a meaningful teaching and learning context for both actors. We can contribute to this through the construction and validation of instruments that relate to constructs and variables associated with tutorial activity in higher education, as well as by transferring information deriving from other international settings that have long experience of implementing university tutoring. 


\section{References}

Álvarez González, M. (2013). Hacia un modelo integral de la tutoría universitaria. In M. Álvarez González \& R. Bisquerra (Coords.), Manual de orientación y tutoría [CD]. Barcelona: Wolters Kluwer España.

Álvarez González, M. (2014). La orientación y la tutoría universitaria en la formación de los grados y postgrados. Las transiciones académicas. In P. Figuera (Coord.), Persistir con éxito en la Universidad: De la investigación a la acción (pp. 175-203). Barcelona: Laertes.

Álvarez González, M. (2017). Hacia un modelo integrador de la tutoría en los diferentes niveles educativos. Educatio Siglo XXI, 35 (2), 21-42.

Álvarez González, M., \& Álvarez, J. (2015). La tutoría universitaria: del modelo actual a un modelo integral. Revista Electrónica Interuniversitaria de Formación del Profesorado, 18 (2), 125-142. doi: http://dx.doi.org/10.6018/reifop.18.2.219671

Álvarez González, M., \& Bisquerra, R. (2012). Orientación educativa. Modelos, áreas estrategias y recursos. Madrid: Wolters Kluwer España.

Álvarez-Pérez, P. R. (2014). La función tutorial del profesorado universitario: Una nueva competencia de la labor docente en el contexto del EEES. Revista Portuguesa de Pedagogía, 47 (2), 85-106.

Álvarez-Pérez, P. R., López, D., \& Pérez, D. (2016). Programa de tutoría universitaria formativa y desarrollo del proyecto personal del alumnado. Revista de Pedagogía, 37 (100), 67-89.

Amor Almedina, M. (2016). Evaluación de la orientación y la tutoría en la Facultad de Ciencias de la Educación de la Universidad de Córdoba. Educatio Siglo XXI, 34 (1), 93-11.

Arza, N., Salvador, X., \& Mascarenhas, S. (2014). La función tutorial del profesorado: Estudio evaluativo en tres universidades federales brasileñas. Revista Electrónica Interuniversitaria de Formación del profesorado, 17 (3), 105-121. Retrieved from https://bit.ly/2W0Rvr0 (Consulted on 2018-12-03).

Bisquerra, R. (2013). Orientación, tutoría y educación emocional. Madrid: Síntesis.
Cashmore, A., Scott, J., \& Cane, C. (2012). «Belonging» and «intimacy» factors in the retention of students an investigation into the student perceptions of effective practice and how that practice can be replicated. Leicester: Universidad de Leicester.

Cid, A., \& Pérez, A. (2006). La tutoría en la Universidad de Vigo según la opinión de sus profesores. $R e$ vista de Investigación Educativa, 24 (2), 395-421.

Da Re, L., \& Clerici, R. (2017). Abandono, rendimiento académico y tutoría. Una investigación en la Universidad de Padua. Educatio Siglo XX1, 35 (2), 139-160.

Echeverría, B. (2002). Gestión de la competencia de acción profesional. Revista de Investigación Educativa, 20 (1), 7-43.

Esteban, M., Bernardo, A. B., \& Rodríguez-Muñiz, L. J. (2016). Permanencia en la universidad: la importancia de un buen comienzo. Aula Abierta, 44, 1-6. doi: https://doi.org/10.1016/j. aula.2015.04.001

Fernández-Salinero, C. (2014). La tutoría universitaria en el EEES: Perfiles actuales. Teoría de la Educación, 26, 161-186. doi: http://dx.doi. org/10.14201/teoredu2014261161186

Figuera, P., \& González, M. Á. (2014). La intervención orientadora y tutorial en la adaptación y persistencia del alumnado en la universidad. Revista de orientación educacional, 28 (54), 31-50.

Gaitán, P. (2013).Hacia una definición de tutoría universitaria. Didac, 14 (61), 4-8. Retrieved from https://bit.ly/31ES4rD (Consulted on 2018-11-24).

García Cabrero, B., Ponce Ceballos, S., García Vigil, M. H., Caso Niebla, J., Morales Garduño, C., Martínez Soto, Y., ... Aceves Villanueva, Y. (2016). Las competencias del tutor universitario: una aproximación a su definición desde la perspectiva teórica y de la experiencia de sus actores. Perfiles educativos, 38 (151), 104-122.

García Nieto, N. (2011). La función tutorial en el ámbito educativo. Padres y Maestros, 342, 5-9.

García Nieto, N., Asensio Muñoz, I., Carballo Santaolalla, R., García García, M., \& Guardia González, S. (2004). Guía para la labor tutorial en la Universidad en el Espacio Europeo de Educación Superior. Madrid: MECD. 
García, M., Carpintero, E., Biencinto, C., \& Núñez, M. C. (2014). La evaluación del proyecto SOU-estuTUtor: percepción de los mentores como punto de referencia para la mejora de un programa de mentoría universitaria para estudiantes de primero. Revista Complutense de Educación, 25 (2), 433-455.

García-Ros, R., Pérez-González, F., Pérez-Blasco, J., \& Natividad, L. (2012). Evaluación del estrés académico en estudiantes de nueva incorporación a la universidad. Revista Latinoamericana de Psicología, 44 (2), 143-154. Retrieved from https:// bit.ly/2BwzOq7 (Consulted on 2018-12-20).

García-Valcárcel, A. (2008). La tutoría en la enseñanza universitaria y la contribución de las TIC para su mejora. RELIEVE, 14 (2), 1-14. Retrieved from https://bit.ly/2JakwLI

Giner, A. (2012). Perfil competencial del tutor o de la tutora de enseñanza secundaria. Revista Española de Orientación y Psicopedagogía, 23 (2), 22-41. doi: https://doi.org/10.5944/reop.vol.23. num.2.2012.11416

González-Benito, A., \& Vélaz de Medrano, C. (2014). La acción tutorial en el sistema escolar. Madrid: UNED.

González-Lorente, C., \& González-Morga, N. (2015). Enseñar a transitar desde la Educación Primaria: el proyecto profesional y vital. Revista Electrónica Interuniversitaria de Formación del profesorado, 18 (2), 29-41. doi: https://doi. org/10.6018/reifop.18.2.219291

Hagenauer, G., \& Volet, S. E. (2014). Teacher-student relationship at university: an important yet under-researched field. Oxford Review of Education, 40 (3), 370-388.

Haya, I., Calvo, A., \& Rodríguez, C. (2013). La dimensión personal de la tutoría universitaria: Una investigación cualitativa en la Universidad de Cantabria (España). Revista Española de Orientación y Psicopedagogía, 24 (3), 98113. doi: https://doi.org/10.5944/reop.vol.24. num.3.2013.11247

Herrera Rodríguez, M. G. (2017). Las tutorías, un apoyo trascendente para los universitarios. $R e-$ vista Iberoamericana de Producción Académica y Gestión Educativa, 7 (4), 1-12. Retrieved from https://bit.ly/2Pf88hc (Consulted on 2018-11-24).
Lázaro, A. (2003). Competencias tutoriales en la universidad. In F. Michavilla \& J. García (Eds.), La tutoría y los nuevos modos de aprendizaje en la universidad (pp. 107-128). Madrid: CAM-Cátedra UNESCO.

León, V. (2018). Funcionamiento de la acción tutorial y factores de eficacia (Doctoral dissertation). Universidad Complutense de Madrid, Madrid.

Lobato, C., \& Guerra, N. (2016). La tutoría en la educación superior en Iberoamérica: Avances y desafíos. Revista Educar, 52 (2), 379-398. doi: https://doi.org/10.5565/rev/educar.726

López-Gómez, E. (2015). La tutoría en el EEES: propuesta, validación y valoración de un modelo integral (Doctoral dissertation). Institutional Repository of the Universidad Nacional de Educación a Distancia, Madrid. Retrieved from https://bit.ly/2JbCPAe

López-Gómez, E. (2017). El concepto y las finalidades de la tutoría universitaria. Una consulta a expertos. Revista Española de Orientación y Psicopedagogía, 28 (2), 61-78. doi: https://doi. org/10.5944/reop.vol.28.num.2.2017.20119

López, I., \& González, P. (2018). La tutoría universitaria como espacio de relación personal. Un estudio de caso múltiple. Revista de Investigación Educativa, 36 (2), 381-399. doi: http:// dx.doi.org/10.6018/rie.36.2.291161

López, I., González, P., \& Velasco, P. J. (2013). Ser y Ejercer de tutor en la universidad. Revista de Docencia Universitaria, 11 (2), 107-134.

Lorenzo, M., Argos, J., Hernández García, J., \& Vera Vila, J. (2013). El acceso y la entrada del estudiante a la universidad: situación y propuestas de mejora facilitadoras del tránsito. Educación XX1, 17 (1), 15-38.

Manzano-Soto, N., \& Roldán-Morales, C. (2015). Análisis de necesidades de orientación del estudiante de primer año en la universidad autónoma de occidente. Revista Española de Orientación y Psicopedagogía, 26 (3), 121140. doi: https://doi.org/10.5944/reop.vol.26. num.3.2015.16404

Martínez-Mínguez, L., \& Moya, L. (2017). La opinión de los estudiantes: la tutoría académica en la adquisición de competencias profesionales de educación física en los graduados en educación infantil. DIDACTICAE, 2, 71-88. 
Martínez Clares, P., Martínez Juárez, M., \& Pérez Cusó, J. (2014). Tutoría Universitaria: entorno emergente en la Universidad Europea. Un estudio en la Facultad de Educación de la Universidad de Murcia. Revista de Investigación Educativa, 32 (1), 111-138.

Martínez Clares, P., Martínez Juárez, M., \& Pérez Cusó, J. (2016). ¿Cómo avanzar en la tutoría universitaria? Estrategias de acción: los estudiantes tienen la palabra. Revista Española de Orientación y Psicopedagogía, 27 (2), 8098. doi: https://doi.org/10.5944/reop.vol.27. num.2.2016.17115

Martínez Clares, P., Pérez Cusó, F. J., \& González Morga, N. (2019). ¿Qué necesita el alumnado de la tutoría universitaria? Validación de un instrumento de medida a través de un análisis multivariante. Educación XX1, 22 (1), 189-213. doi: 10.5944/educXX1.21302

Más Torelló, Ó. (2012). Las competencias del docente universitario: la percepción del alumno, de los expertos y del propio protagonista. Revista de Docencia Universitaria, 10 (2), 299318.

McFarlane, K. J. (2016). Tutoring the tutors: supporting effective personal tutoring. Active Learning in Higher Education, 17 (1), 77-88. doi: https://doi. org $/ 10.1177 / 1469787415616720$

Pantoja, A. (2013). La acción tutorial en la escuela. Madrid: Editorial Síntesis.

Pérez, F. J., González, C., González, N., \& Martínez, M. (2017). Tutoría en la Universidad: un estudio de caso en la Facultad de Educación de la Universidad de Murcia. Educatio Siglo XXI, 2 (35), 91-110.

Pujol, E., \& Durán, E. (2013). Manejo del tiempo académico en jóvenes que inician estudios en la Universidad Simón Bolívar. Latinoamericana de Ciencia Sociales, Niñez y Juventud, 11 (1), 93-108.

Royal Decree 1791/2010, of December 30, by which the Statute of the University Student is approved. Official State Gazette of Spain, No. 318, 31 December 2010, pp. 109353 a 109380. Retrieved from https://www.boe.es/boe/dias/2010/12/31/ pdfs/BOE-A-2010-20147.pdf (Consulted on 2019-10-22).
Rebollo, N., \& Espiñeira, E. M. (2017). La tutoría durante el proceso de desarrollo del TFG y TFM: análisis del grado de utilidad y satisfacción del alumnado. Educatio Siglo XXI, 35 (2), 161-180.

Rodríguez Espinar, S. (2004). Manual de tutoría universitaria. Recursos para la acción. Barcelona: Octaedro-ICE.

Soares, A. P. C., Almeida, L. S., \& Guisande, M. A. (2011). Ambiente académico y adaptación a la universidad: un estudio con estudiantes de $1^{0}$ año de la Universidad do Minho. Revista iberoamericana de psicología y salud, 2 (1), 99-121. Retrieved from https://bit. $\mathrm{ly} / 33 \mathrm{~W} 20 \mathrm{Nt}$

Solaguren-Beascoa, M., \& Moreno, L. (2016). Escala de actitudes de los estudiantes universitarios hacia las tutorías académicas. Educación XX1, 19 (1), 247-266. doi: https://doi.org/10.5944/ educxx1.15586

Torrecilla, E. M., Rodríguez, M. J., Herrera, M. E., \& Martín, J. F. (2013). Evaluación de calidad de un proceso de tutoría de titulación universitaria: La perspectiva del estudiante de nuevo ingreso en educación. Revista Española de Orientación y Psicopedagogía, 24 (2), 79-99. doi: https://doi.org/10.5944/reop.vol.24. num.2.2013.11260

Troyano, Y., \& García, A. J. (2009). Expectativas del alumnado sobre el profesorado tutor en el contexto del Espacio Europeo de Educación Superior. Revista Docencia Universitaria, 3, 1-8. Retrieved from https://bit.ly/2J74XEH

Urbina, A., de la Calleja, J., \& Medina, M. A. (2017). Associating students and teachers for tutoring in higher education using clustering and data mining. Computers Applications in Engineering Education, 25 (5), 823-832.

Waltz, S. B. (2019). Tutor training for service learning: impact on self-efficacy beliefs. Mentoring \& Tutoring: Partnership in Learning, 27 (1), 26-43. doi: 10.1080/13611267.2019.15 83405

Yale, A. T. (2019). The personal tutor-student relationship: student expectations and experiences of personal tutoring in higher education. Journal of Furtherand HigherEducation, 43 (4), 533544. doi: 10.1080/0309877X.2017.1377164 


\section{Authors' biographies}

Manuel Delgado-García holds a Degree and Doctorate in Educational Psychology from the Universidad de Huelva, and a Degree in Pedagogy from the Universidad Nacional de Educación a Distancia (UNED). He is Assistant Professor in the Department of Pedagogy of the Universidad de Huelva and collaborator with the Universidad Internacional de la Rioja (UNIR). His research interests focus on orientation, tutorial activity, and teachers' professional development.

(iD https://orcid.org/0000-0001-6157-0494

Sara Conde Vélez holds a Degree and Doctorate in Educational Psychology from the Universidad de Huelva. She is Assistant Professor in the Department of Art and Architecture of the Universidad de Huelva. Her main research interests revolve around school relations, school bullying, and the design and validation of evaluation instruments.

iD https://orcid.org/0000-0002-7950-5866
Ángel Boza Carreño holds a Degree in Education and Doctorate in Educational Psychology from the Universidad de Huelva. Educational guidance counsellor (19831997) and Associate Professor in the Research and Diagnosis Methods in Education Area, which forms part of the Department of Pedagogy of the Universidad de Huelva. His research interests focus on educational orientation and tutorial activity as well as research methods in education.

iD https://orcid.org/0000-0002-3395-421X 


\section{revista española de pedagogía año 78, n 275, enero-abril 2020 \\ Spanish Journal of Pedagogy \\ year 78, n. 275, January-April 2020 \\ Table of Contents \\ Sumario}

\section{Mathematics teaching} issues

Cuestiones de enseñanza de las matemáticas

Wendolyn Elizabeth Aguilar-Salinas, Maximiliano de las Fuentes-Lara, Araceli Celina Justo-López, \& Ana Dolores Martínez-Molina

A measurement instrument for establishing the algebraic skills of engineering students on a Differential Calculus Course in engineering Instrumento de medición para diagnosticar las habilidades algebraicas de los estudiantes en el Curso de Cálculo Diferencial en ingeniería

Maria Burgos, Pablo Beltrán-Pellicer, \& Juan D. Godino The issue of didactical suitability in mathematics educational videos: experience of analysis with prospective primary school teachers La cuestión de la idoneidad de los vídeos educativos de matemáticas: una experiencia de análisis con futuros maestros de educación primaria

27
Online training in the world of education: experiences from the United States La formación online en el mundo de la educación: experiencias de los Estados Unidos

Gerald LeTendre, \& Tiffany Squires

Integrating online and residential master's programs in education

Integración de programas de máster online y presenciales en educación

\section{Laurence B. Boggess}

Innovations in online faculty development: an organizational model for long.term support of online faculty

Innovación en la capacitación docente online: un modelo organizacional para brindar apoyo a largo plazo a la docencia online

Brian Redmond

Shared team leadership for an online program Liderazgo de equipo compartido de un programa online 
Studies and Notes

Estudios y Notas

\section{Paolo Scotton}

Thinking together, living fully. Experiencing philosophy

with children

Pensar en común, vivir en plenitud. La experiencia de la

filosofía con los niños y las niñas

103

Manuel Delgado-Garcia, Sara Conde Vélez, \& Ángel

\section{Boza Carreño}

Profiles and functions of university tutors and their

effects on students' tutorial needs

Perfiles y funciones del tutor universitario y sus efectos sobre

las necesidades tutoriales del alumnado

119
Book reviews

Luri, G. (2019). La imaginación conservadora: una defensa apasionada de las ideas que han hecho del mundo un lugar mejor [The conservative imagination: A passionate defence of ideas that have made the world a better place] (Enrique Alonso Sainz). Quigley, C. F., \& Herro, D. (2019). An educator's guide to STEAM. Engaging students using real-world problems (Juan Luis Fuentes). Prince, T. (2019). Ejercicios de mindfulness en el aula. 100 ideas prácticas [Mindfulness exercises for the class. 100 practical ideas] (José V. Merino Fernández).

145

\section{Call for papers}

Solicitud de originales

This is the English version of the research articles and book reviews published originally in the Spanish printed version of issue 275 of the revista española de pedagogía. The full Spanish version of this issue can also be found on the journal's website http://revistadepedagogia.org.

ISSN: 0034-9461 (Print), 2174-0909 (Online)

https://revistadepedagogia.org/

Depósito legal: M. 6.020 - 1958

INDUSTRIA GRÁFICA ANZOS, S.L. Fuenlabrada - Madrid 